\title{
A molecular insight into the dissociable regulation of associative learning and motivation by the synaptic protein neuroligin-1
}

Jiaqi Luo, Jessica M. Tan and Jess Nithianantharajah ${ }^{*}$ (1)

\begin{abstract}
Background: In a changing environment, a challenge for the brain is to flexibly guide adaptive behavior towards survival. Complex behavior and the underlying neural computations emerge from the structural components of the brain across many levels: circuits, cells, and ultimately the signaling complex of proteins at synapses. In line with this logic, dynamic modification of synaptic strength or synaptic plasticity is widely considered the cellular level implementation for adaptive behavior such as learning and memory. Predominantly expressed at excitatory synapses, the postsynaptic cell-adhesion molecule neuroligin-1 (Nlgn1) forms trans-synaptic complexes with presynaptic neurexins. Extensive evidence supports that Nlgn1 is essential for NMDA receptor transmission and long-term potentiation (LTP), both of which are putative synaptic mechanisms underlying learning and memory. Here, employing a comprehensive battery of touchscreen-based cognitive assays, we asked whether impaired NMDA receptor transmission and LTP in mice lacking Nlgn1 does in fact disrupt decision-making. To this end, we addressed two key decision problems: (i) the ability to learn and exploit the associative structure of the environment and (ii) balancing the trade-off between potential rewards and costs, or positive and negative utilities of available actions.
\end{abstract}

Results: We found that the capacity to acquire complex associative structures and adjust learned associations was intact. However, loss of Nlgn1 alters motivation leading to a reduced willingness to overcome effort cost for reward and an increased willingness to exert effort to escape an aversive situation. We suggest Nlgn1 may be important for balancing the weighting on positive and negative utilities in reward-cost trade-off.

Conclusions: Our findings update canonical views of this key synaptic molecule in behavior and suggest Nlgn1 may be essential for regulating distinct cognitive processes underlying action selection. Our data demonstrate that learning and motivational computations can be dissociated within the same animal model, from a detailed behavioral dissection. Further, these results highlight the complexities in mapping synaptic mechanisms to their behavioral consequences, and the future challenge to elucidate how complex behavior emerges through different levels of neural hardware.

Keywords: Cost-benefit trade-off, Learning and memory, Motivation, NL1, Response latency, Response vigor, Rodent touchscreens

\footnotetext{
* Correspondence: jess.n@florey.edu.au

Florey Institute of Neuroscience and Mental Health, Florey Department of Neuroscience, Melbourne Brain Centre, University of Melbourne, 30 Royal Parade, Parkville, Victoria 3052, Australia
}

(c) The Author(s). 2020 Open Access This article is licensed under a Creative Commons Attribution 4.0 International License, which permits use, sharing, adaptation, distribution and reproduction in any medium or format, as long as you give appropriate credit to the original author(s) and the source, provide a link to the Creative Commons licence, and indicate if changes were made. The images or other third party material in this article are included in the article's Creative Commons licence, unless indicated otherwise in a credit line to the material. If material is not included in the article's Creative Commons licence and your intended use is not permitted by statutory regulation or exceeds the permitted use, you will need to obtain permission directly from the copyright holder. To view a copy of this licence, visit http://creativecommons.org/licenses/by/4.0/ The Creative Commons Public Domain Dedication waiver (http://creativecommons.org/publicdomain/zero/1.0/) applies to the data made available in this article, unless otherwise stated in a credit line to the data. 


\section{Background}

In a changing environment, a challenge for the brain is to adaptively guide behavior towards survival which involves the processing of sensory information, selecting between actions that will most likely result in a beneficial outcome, and executing these actions. These complex cognitive abilities emerge from the physical architecture of the brain: from circuits to neurons, synapses, and ultimately the molecular components that comprise the protein signaling complexes at synaptic terminals. This hardware forms the basis to enable signaling and plasticity within and between brain regions, thus supporting the emergence of behavior. In line with this logic, intact synaptic transmission and plasticity are widely held as the key cellular level mechanisms required for adaptive behavior such as learning and memory. At the postsynaptic density (PSD) of excitatory synapses, neuroligin-1 (Nlgn1) is a cell-adhesion molecule that binds presynaptic neurexins to form transsynaptic complexes [1, 2]. Aligning PSD components with presynaptic neurotransmitter release sites [3-5], Nlgn1 directly binds postsynaptic scaffolds including PSD-95 [3] and promotes retention of $\alpha$-amino-3-hydroxyl-5-methyl-4-isoxazole-propionate (AMPA) and $N$ methyl-D-aspartate (NMDA) receptors by indirect intracellular and direct extracellular interactions in developing and mature synapses [6-9].

Importantly, Nlgn1 is well established to be required for intact NMDA receptor function and long-term potentiation (LTP), both of which are the key synaptic mechanisms thought to underlie learning and memory [10-13]. NMDA receptor-mediated postsynaptic currents have been consistently shown to be decreased across several brain regions by Nlgn1 knockout or knockdown [7, 14-23] and, conversely, increased by Nlgn1 overexpression [22-24]. Further, Nlgn1 has been repeatedly shown to be essential for synaptic plasticity and the induction of both NMDA receptor-dependent and independent LTP in multiple brain regions $[7,14$, 15, 17-20, 25, 26]. Therefore, a reasonable assumption may be that Nlgn1 is also important for learning and memory processes. Indeed, Blundell and colleagues reported disrupted grooming behavior and spatial learning and memory in the Morris water maze in mice lacking Nlgn1 [14]. This is further supported by impaired performance in the water maze in Nlgn1 overexpression mouse models [24, 27] and contextual and cued fear memory recall deficits in rats with basal lateral amygdala knockdown of Nlgn1 [19], collectively supporting the idea that Nlgn1 is necessary for learning and memory. In contrast to the extensive molecular and physiological characterization of Nlgn1, only these few studies have examined Nlgn1 in behavior, and specifically none has evaluated different components of complex cognitive behavior in detail. Thus, whether impaired NMDA receptor function and synaptic plasticity caused by loss of Nlgn1 translates to impact all forms of learning and the wider cognitive repertoire is unclear.

In this study, employing a comprehensive battery of touchscreen-based cognitive assays, we sought to assess male and female mice lacking Nlgn1 on two key decision problems: (1) the ability to learn and exploit the associative structure of the environment and (2) balancing the trade-off between potential rewards and costs, or positive and negative utilities associated with available actions. We found that mice lacking Nlgn1 have an intact capacity to acquire complex associative structures and adjust learned associations. However, loss of Nlgn1 alters motivation leading to a reduced willingness to overcome response effort for reward and increased willingness to exert effort to escape an aversive situation. We suggest these divergent phenotypes may converge on a model of increased weighting on negative utilities, highlighting a novel valence-dependent role of Nlgn1 in balancing the weighting on positive and negative utilities in rewardcost trade-off. Our data identify unexpected findings that update current views of this key synaptic molecule to show Nlgn1 is essential for regulating distinct cognitive processes underlying decision-making. Our findings demonstrate that learning and motivational computations can be behaviorally dissected, contributing to unraveling the genetic architecture of dissociable cognitive modules. Further, these behavioral findings highlight the complexity in directly mapping synaptic mechanisms to their behavioral consequences, thus the future challenge and importance of elucidating how complex behavior emerges through different levels of neural hardware.

\section{Results}

Nlgn1 is not essential for learning complex associative structures

Intact long-term forms of plasticity and NMDA receptor function are both synaptic mechanisms thought to be required for learning and memory (e.g., [13, 28-35]). Based on the established impairments in NMDA receptor function and LTP combined with the previous behavioral reports, we hypothesized that Nlgn1 is likely to be important for acquiring associative structures of the environment, and using these structures to optimize action selection. To address this, we assessed male and female null mutant mice lacking $\operatorname{Nlgn} 1\left(N \operatorname{lgn} 1^{-1-}\right)$ and control wildtype (WT) littermates in a series of rodent touchscreen cognitive tests, where mice were required to make responses via nose-pokes to different visual stimuli displayed on a touchscreen to obtain rewards under different test situations. Of note, across all the cognitive tests and analyses performed, we observed no significant interactions between genotype and sex (except for that 
presented in Fig. 4f, data in Additional file 1: Fig. S13); therefore, combined data for both sexes by genotype will be presented for clarity. Additionally, we employed trialby-trial analyses that better describe complex behavioral data (see the "Methods" section and Additional file 2: Table S1 for detailed statistical results).

Animals first underwent several phases of instrumental training (touchscreen pre-training) to learn to initiate the commencement of trials and selectively nose-poke simple visual stimuli displayed on the touchscreen in order to obtain a liquid reward (strawberry milk) [36, 37]. $\mathrm{NlgnI}^{-1-}$ and WT mice required similar numbers of sessions to complete the pre-training phases, indicating loss of Nlgn1 does not impact the acquisition of simple instrumental conditioning (Additional file 1: Fig. S2). Following pre-training, mice were introduced to the pairwise visual discrimination task, a forced choice paradigm where two visually similar stimuli were presented pseudorandomly between two locations (left or right side of the touchscreen). Responses to one of the stimuli were rewarded $(\mathrm{S}+)$ while responses to the other were unrewarded (S-) (Fig. 1a). The visual discrimination task therefore requires mice to learn to perceptually discriminate the stimuli and selectively respond to the correct or rewarded stimulus regardless of stimulus location. Performance accuracy (percentage of correct responses) was the primary measure used to track learning across training sessions, until a learning criterion was reached. We found that $\mathrm{NlgnI}^{-/-}$mice required similar numbers of trials to reach the discrimination learning criterion as WT controls (Fig. 1b). Similarly, the percentage of $N \operatorname{lgn} 1^{-1-}$ and WT mice reaching criterion across sessions was not different (Fig. 1b). To understand how key variables including genotype, sex, and session affect response accuracy, we estimated the effect of these variables on trial outcomes (correct/incorrect responses) using a mixed-effect generalized linear model [38]. We observed a highly significant effect of session as expected, reflecting the improvements on response accuracy over sessions. However, there was no effect of genotype on trial outcomes (correct responding of $N \operatorname{lgn} 1^{-1-}$ relative to WT expressed as odds ratio, Fig. 1c) nor a significant genotype $\times$ session interaction, indicating that both $N \operatorname{lgn} 1^{-1-}$ and WT mice acquired visual discrimination learning at a similar rate (Fig. 1c), consistent with our trials to learning criterion and percentage of mice reaching criterion analyses. Furthermore, these data combined with the normal instrumental conditioning (pre-training) also confirm loss of Nlgn1 does not impair basic perceptual processing abilities.

To increase demands on associative complexity by having to integrate both visual and spatial information as features defining the reward contingencies, we employed the object-location paired associate learning task which requires mice to learn not only the perceptual features of three stimuli (flower, plane, spider) but also their unique rewarded location on the touchscreen (left, center, right respectively). On each trial, only two stimuli are presented: one displayed in its correct location $\left(\mathrm{S}_{+}\right)$and the other in an incorrect location (S-) (Fig. 1d). Compared to the visual discrimination task, the greater difficulty in acquiring visuospatial associations in this object-location paired associate learning task is reflected in a slower rate of learning, evident by the increased number of training sessions (Fig. 1e) and smaller effect size of session (Fig. 1f). Despite this increased difficulty, we again observed $N \operatorname{lgn} 1^{-/-}$mice were able to acquire the complex object-location associations similar to WT mice (Fig. 1e, f). There were subtle suggestions that $N \operatorname{lgn} 1^{-1-}$ mice may have reduced accuracy in later sessions ( 25 onwards), but since there was no significant genotype $\times$ session interaction $(p=0.1$, Additional file 2: Table S1), we deemed it inappropriate to follow up further analyses separating out stages of paired associate learning.

\section{Flexible updating of learned associations is not altered by loss of Nlgn1}

Adapting to dynamic environments where the outcome of a response is not always stable requires the ability to inhibit learned responses once they no longer yield positive outcomes, and explore alternatives. To examine the requirement of Nlgn1 for flexible adjustment of response selection, we employed two tests: reversal and extinction learning. Both these tests probe flexible responding but can depend on distinct genetic and neural basis (e.g., [39]). First, we examined cognitive flexibility in a test of reversal learning. Once mice had reached the learning criterion on pairwise visual discrimination (Fig. 1a-c), we reversed the reward contingencies so that the previously rewarded stimulus was now unrewarded and vice versa (Fig. 2a). All mice displayed a strong tendency to respond to the previously rewarded stimulus (now $\mathrm{S}-$ ) at the beginning of reversal learning, then gradually shifted their responding to the updated $\mathrm{S}_{+}$(previous $\mathrm{S}-$ ) as expected (Fig. 2b). We observed no differences in response accuracy between $\mathrm{NlgnI}^{-1-}$ and WT mice throughout reversal learning (effect of genotype) nor the rate of reversal learning (genotype $\times$ session interaction) (Fig. 2c).

Second, we investigated extinction learning measuring the rate at which mice stop making a learned instrumental response when those responses no longer result in an outcome, and there are no competing operant response alternatives. Mice were first trained to robustly respond to a simple stimulus (white square), and once a stable performance criterion was reached, extinction was tested in which responses to the stimulus were no longer rewarded (Fig. 2d). On each trial, mice could either 


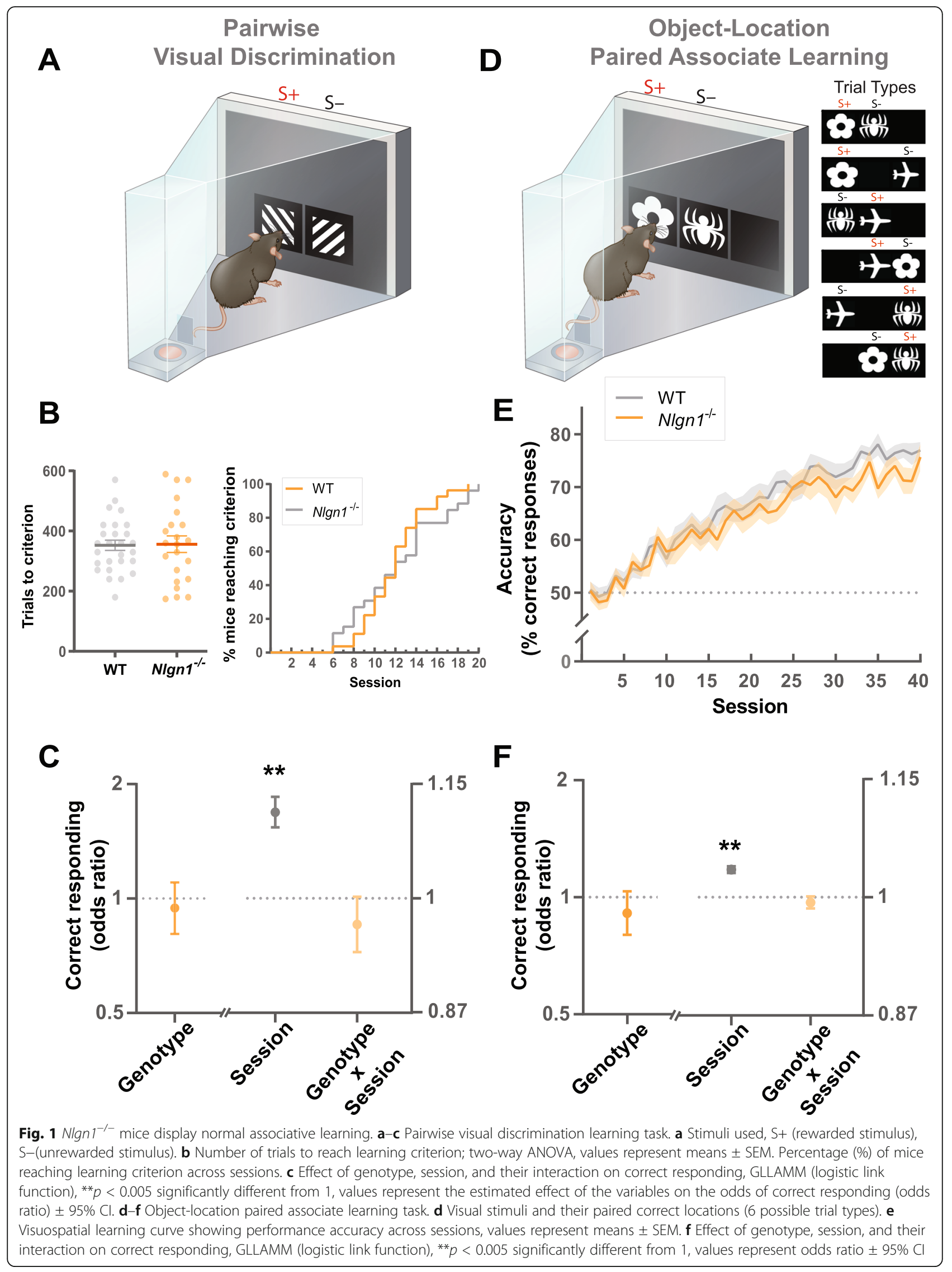


make or omit a response within a set time window. One challenge in quantifying the rate of instrumental extinction is that feedback on reward contingency is only provided after a response. As a result, animals that display slower instrumental extinction for example (therefore made more responses) will have received more learning opportunities due to greater feedback, and vice versa. To minimize potential differences in learning opportunities, we set a limit on the number of trials per session for all animals and assessed extinction learning across sessions (Fig. 2e, Additional file 1: Fig. S3). We observed no difference between $\mathrm{NlgnI}^{-1-}$ and WT mice on whether they responded or omitted a trial throughout extinction (effect of genotype) and rates of extinction both within a session (effect of trial, genotype $\times$ trial) and across sessions (effect of session, genotype $\times$ session) (Fig. 2f). To confirm potential differences in extinction rate was not masked by differences in learning opportunities, we also analyzed the rate of extinction as the effect of $\mathrm{cu}-$ mulative responses and again found no differences (data not shown) indicating intact instrumental extinction learning.

Previous work has reported that $\mathrm{Nlgn1}^{-1-}$ mice display increased self-grooming [14] thought to represent repetitive and stereotypic behavior common in brain disorders such as autism spectrum disorder and obsessive-compulsive disorder [40, 41]. We were therefore interested to explore measures of repetitive or perseverative response selection across our multiple learning assays. In the pairwise visual discrimination, object-location paired associate learning, and reversal learning tasks, a correct response to a firstpresentation pseudorandom trial (referred to as a "trial") was always followed by another trial, where the stimuli and location are displayed in a pseudorandom and counterbalanced manner. In contrast, an incorrect response was always followed by a "correction trial" where the exact same stimulus-location configuration of that (pseudorandom) trial is repeatedly presented until mice switch their response to make a correct response and earn a reward (Additional file 1: Fig. S4A). A perseveration index (PI) calculated as the average number of correction trials committed per incorrect response has been commonly used to measure perseverative responding (e.g., [35, 36, 42]). However, to more explicitly and quantitatively examine perseverative responding on correction trials, we estimated the effect of correction trials on correct responding. Mice were less accurate on correction trials suggesting a tendency to reselect the same incorrect response previously selected (Fig. 2g, Additional file 1: Fig. S5). Consistent with a tendency of repeating the previous response, mice were more accurate when the same stimulus-location configuration happened to reoccur on a consecutive trial (referred to as a "reoccurring trial"), therefore more likely to reselect a correct response previously selected (Fig. 2g, Additional file 1: Fig. S5). However, we found no differences in perseverative responding between $\mathrm{Nlgn}^{-1-}$ and WT mice (genotype $\times$ correction trial, genotype $\times$ reoccurring trial) (Fig. 2g). These data show mice appear to have a general tendency towards perseverative action selection.

\section{Mice lacking Nlgn1 take longer to perform instrumental actions for rewards}

Decision-making in the natural world involves more than choosing the response with the highest expected rewards. Actions may have very different effort requirements; therefore, balancing the trade-off between rewards and costs is a crucial part of maximizing the net utility of actions. We have so far examined task measures that involve action selection between two alternatives that require the same amount of physical effort and only differ in the expected rewards (e.g., performance accuracy calculated on correct vs incorrect responding). But like most naturalistic decision problems, these touchscreen-based tasks are freeoperant tasks in that mice are free to choose a wide range of other actions (e.g., exploring, resting) instead of choosing to execute actions towards earning a reward (initiating a trial, making a response, collecting a reward). To capture an animal's engagement in performing instrumental actions in our tasks, we analyzed several latency parameters: trial initiation, response, and reward collection (Fig. 3a, see the "Methods" section and Additional file 1: Fig. S4B). Response latency was further separated into( i) stimulus-approach latency: time taken after initiating a trial to reach the front of the chamber near the touchscreen and (ii) stimulus-selection latency: time taken from reaching the front of the chamber near the touchscreen and making a nose-poke response to a stimulus. Dissecting the response latencies revealed stimulus-selection latencies positively predicted performance accuracy suggesting it influences the decision between correct and incorrect responses, whereas stimulus-approach latencies did not, suggesting it reflects the decision between choosing to make a response or not (Additional file 1: Fig. S7). Distribution-wide analysis $(0.05-0.95$ th quantile at 0.05 steps) across all three of our tasks (pairwise visual discrimination, object-location paired associate learning, reversal learning) consistently revealed the same pattern for the various latency measures (Fig. 3). $N \operatorname{lgn} 1^{-1-}$ mice were significantly slower to initiate trials, approach stimuli, and collect rewards (Fig. 3b-d, Additional file 1: Fig. S8). Although differences in 


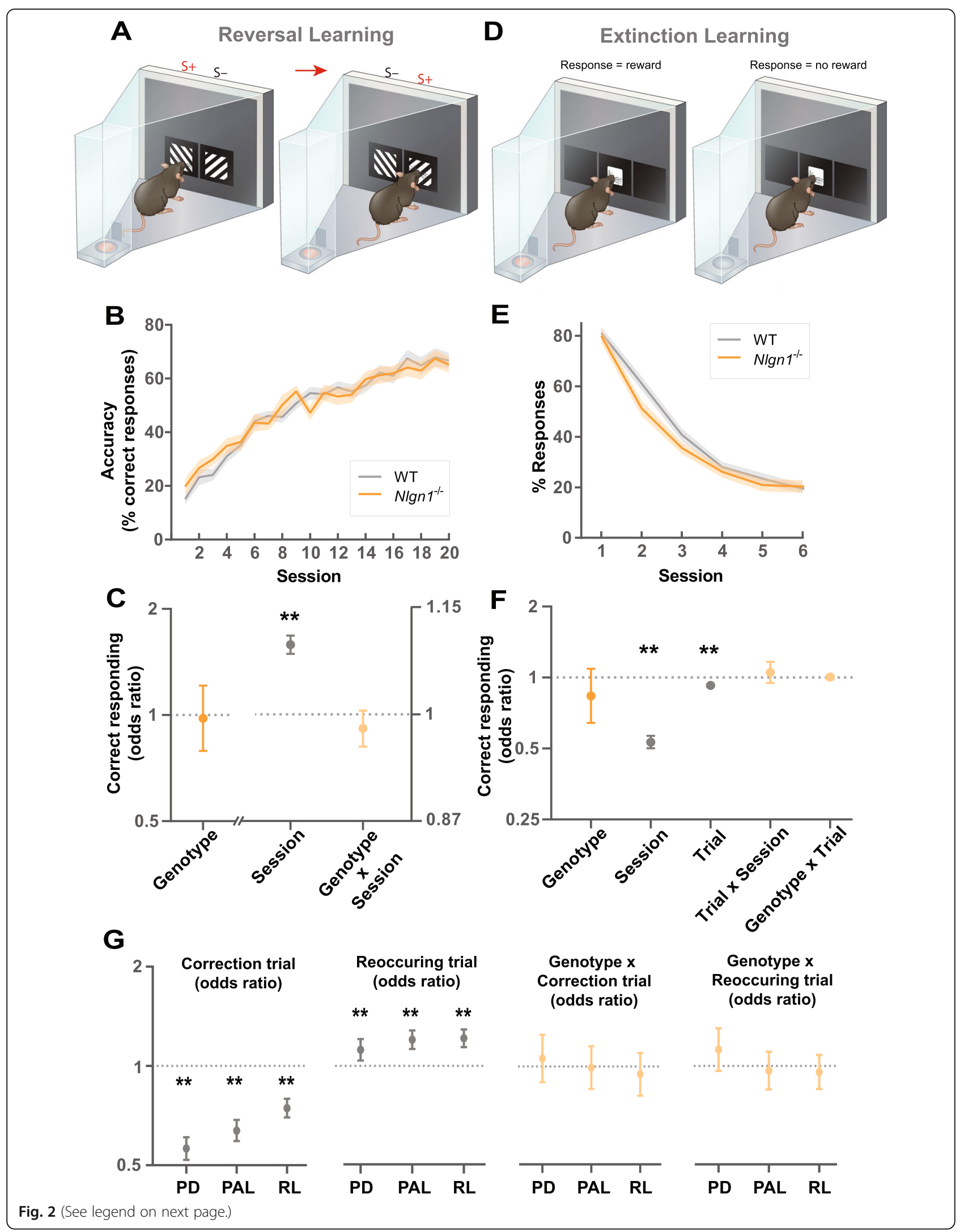


(See figure on previous page.)

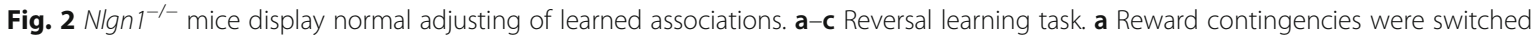
following acquisition of learning criterion for visual discrimination, S+ (rewarded stimulus), S- (unrewarded stimulus). b Reversal learning curve showing performance accuracy across sessions, values represent means \pm SEM. c Effect of genotype, session, and their interaction on correct responding, GLLAMM (logistic link function), ${ }^{* *} p<0.005$ significantly different from 1, values represent odds ratio $\pm 95 \% \mathrm{Cl}$. $\mathbf{d}$-f Extinction learning task. $\mathbf{d}$ Once robust instrumental responding to a performance criterion was reached, responses were no longer rewarded. e Extinction learning curve showing percentages of responses across sessions, values represent means \pm SEM. $\mathbf{f}$ Effect of genotype, session, trial within a session, and their interactions on responding, GLLAMM (logistic link function), ${ }^{* *} p<0.005$ significantly different from 1, values represent the estimated effect of the variables on the odds of correct responding (odds ratio) $\pm 95 \% \mathrm{Cl} . \mathbf{g ~ N} / g n 1^{-1-}$ and WT mice both display similar levels of perseverative behavior in response selection across tasks (pairwise visual discrimination, PD; object-location paired associate learning, PAL; reversal learning, RL). Mice were less accurate on correction trials (effect of correction trial on correct responding, effect sizes $<1$ ); more accurate on pseudorandom trials with reoccurring stimulus configurations (effect of reoccurring pseudorandom trial on correct responding, effect size $>1$ ); but there were no differences due to genotype (effect of correction trial $\times$ genotype interaction, effect of reoccurring pseudorandom trial $\times$ genotype interaction). GLLAMM (logistic link function), ${ }^{* *} p<0.005$, values represent the estimated effect of the variables on the odds of correct responding (odds ratio) $\pm 95 \% \mathrm{Cl}$

median stimulus-approach latency were not statistically significant between genotypes, distribution-wide quantile regressions showed a clear distributional shift towards longer latencies (Fig. 3d). In comparison, stimulus-selection latencies were nearly identical between $\mathrm{Nlgn}^{-1-}$ and WT mice (Fig. 3e).

\section{Loss of Nlgn 1 reduces motivation to overcome response effort for rewards}

Based on the increased latencies $N \operatorname{lgn} 1^{-1-}$ mice displayed, we hypothesized Nlgn1 may be important for regulating specific components of motivational processing. To examine this, we first tested naive mice sequentially across sessions that required a fixed ratio of responding for rewards with increasing demands (FR1-40, Fig. 4a). We wondered whether loss of Nlgn1 might reduce the number of responses at higher ratio requirements where responding has a lower utility, resembling well-characterized models of amotivation [43-45]. Indeed, when the responsereward ratio was low (FR1), $N \operatorname{lgn} 1^{-1-}$ mice performed like WT controls indicating similar motivation to respond when response utility is high, and similar rate to reach satiety. However, as this ratio increased (FR5, FR20, FR40), $\mathrm{Nlgn} 1^{-/-}$mice started to make significantly fewer responses, with the reduction being greater at the higher ratio requirements (Fig. 4b, c, Additional file 1: Fig. S9). This increasing difference in responses between genotypes across ratio requirements was primarily driven by the non-linear increase in the latency taken to re-engage in responding after consuming a reward (post-reinforcement pause, Fig. 4d, Additional file 1: Fig. S10A) and the average time interval between each subsequent response (Fig. 4e, Additional file 1: Fig. S10B, Fig. S11). Next, using a separate naive cohort of mice, we wanted to see if we could observe the same motivational phenotype in a progressive ratio task where ratio requirements progressively increased within a session until mice stop responding (breakpoint). Indeed, we were able to reproduce the same finding with $N \operatorname{lgn} 1^{-/-}$mice making fewer responses and therefore having a lower breakpoint relative to controls (Additional file 1: Fig. S12).

Reduced motivation could be due to taste insensitivity to palatable rewards with higher caloric value (e.g., strawberry milk in this case). To examine whether the observed phenotype in $N \operatorname{lgn} 1^{-/-}$mice was specific to high-fat-high-sugar strawberry milk rewards, we next used water as the reward and measured responding at a fixed ratio where a robust difference was observed using strawberry milk (FR20). Similar to that observed with strawberry milk rewards, Nlgn1 $1^{-1-}$ mice made significantly fewer responses for water rewards (Fig. 4f), with this effect being stronger in females compared to males (Additional file 1: Fig. S13A). Importantly, there were significant positive correlations between the numbers of responses for strawberry milk and water rewards, indicating that mice (either WT or $\mathrm{Nlgn1}^{-{ }^{--}}$) that were more motivated by strawberry milk also responded more for water (Fig. 4g, Additional file 1: Fig. S13B). Together, these data show loss of Nlgn1 impacts instrumental responding motivated by both hunger and thirst.

\section{$\mathrm{Nlgn} 1^{-/-}$mice exert less effort to earn rewards but more effort to escape from aversion}

To investigate the generalizability of the observed motivational phenotype, we wanted to next examine whether $N \operatorname{lgn} 1^{-/-}$mice were also less willing to exert effort outside an operant environment. We assessed exploration and spontaneous locomotor activity in a novel, openfield environment (Fig. 5a) (in darkness) to measure the decision between exploration and resting. We tested two separate cohorts of animals, one group that had previously been tested in an operant paradigm and a second experimentally naive group. Interestingly, in the group that had previous experimental experience, we see that $N \operatorname{lgn} 1^{-1-}$ mice travel a shorter distance (Fig. 5c) and spent more time resting (Fig. 5d) compared to WT controls, consistent with a reduced willingness to overcome the cost of physical effort. We noted that when we 


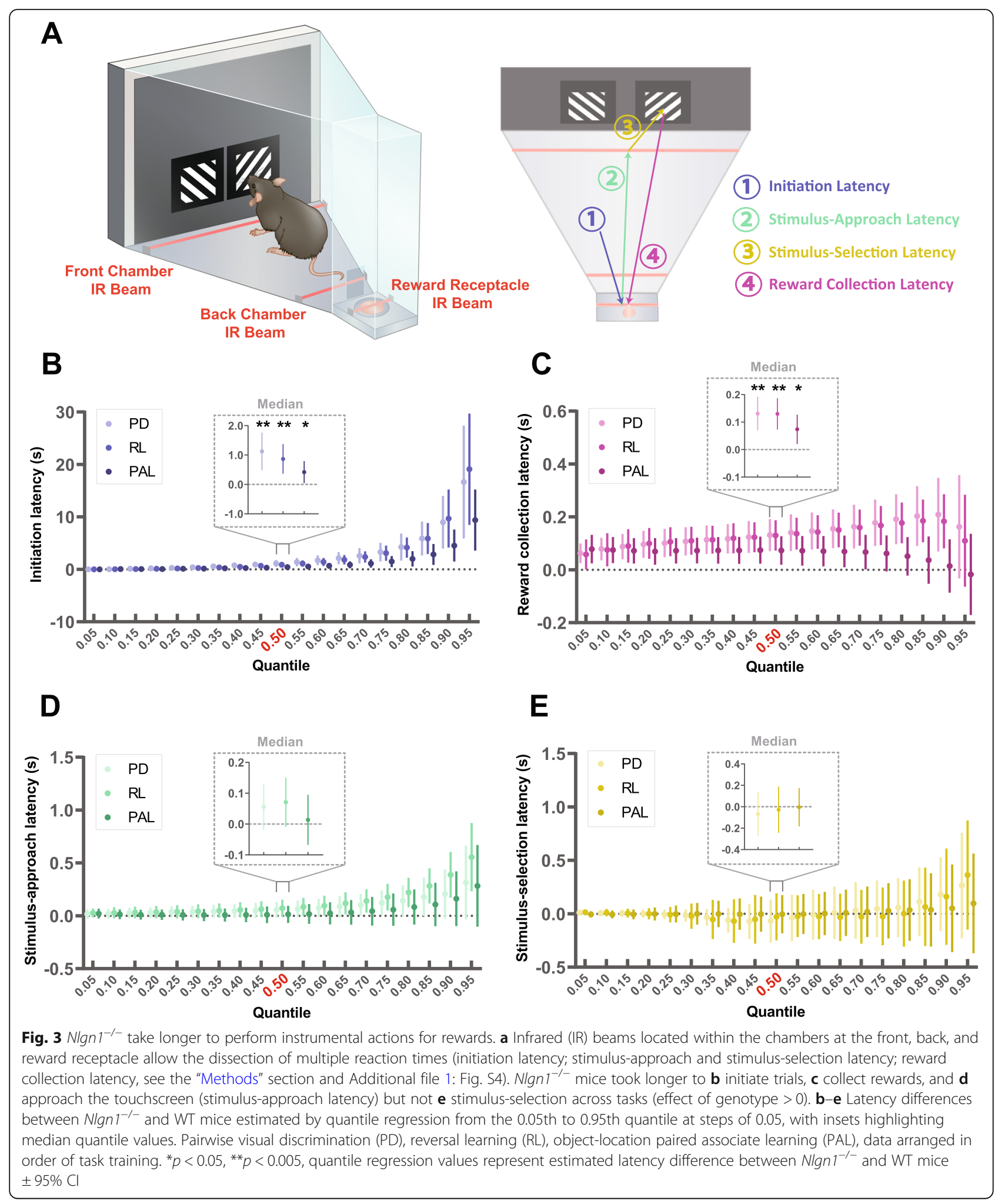

assessed the naive cohort of animals, we did not see a significant effect of genotype (Additional file 1: Fig. S14A-B) suggesting these measures of exploratory behavior are strongly influenced by previous experience.
However, in both groups, movement velocity of $\mathrm{Nlgn}^{-1-}$ mice in the open-field arena was either not different (Fig. 5e) or greater than WT controls (Additional file 1: Fig. S14C). This was further supported by no differences 

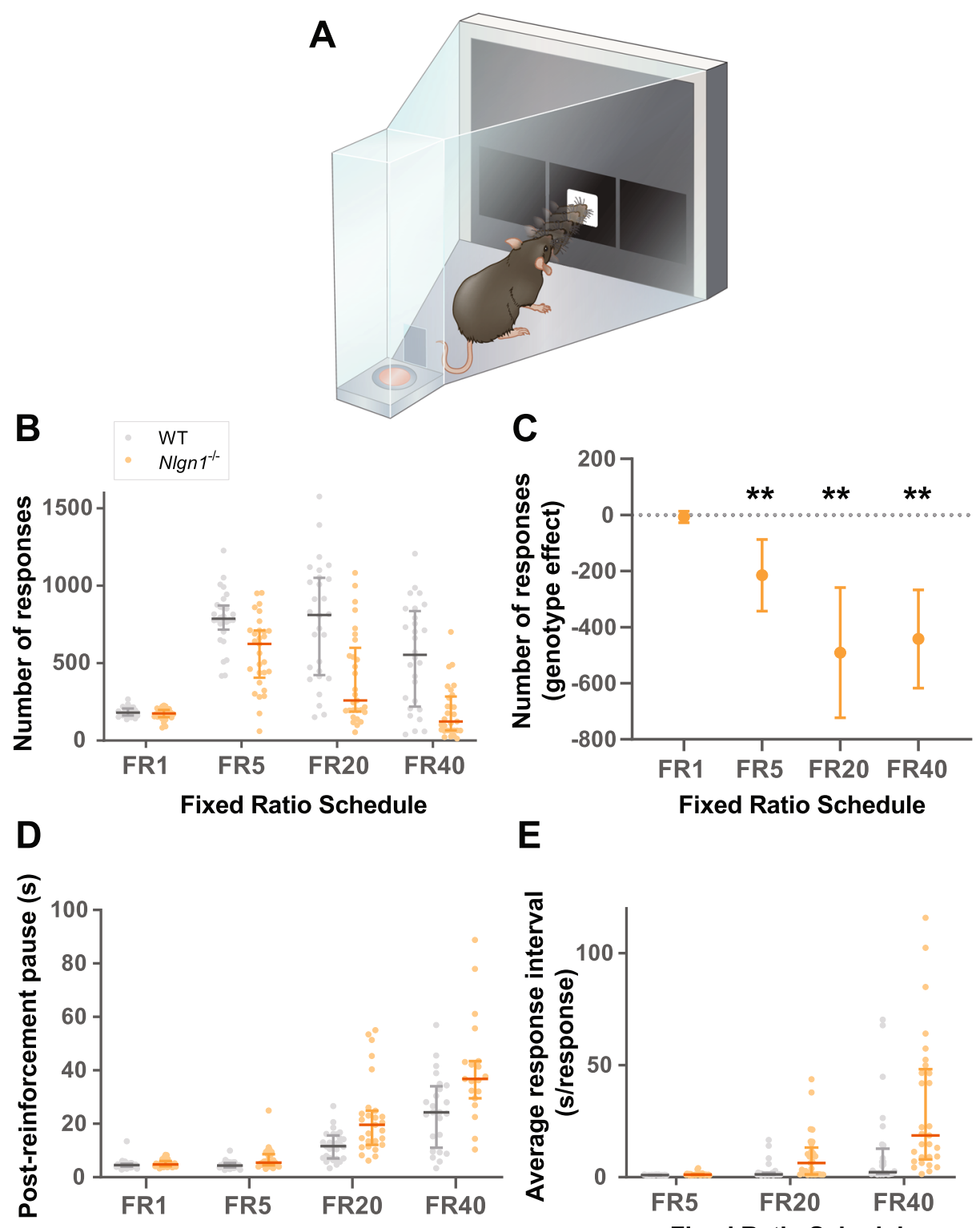

E

Fixed Ratio Schedule

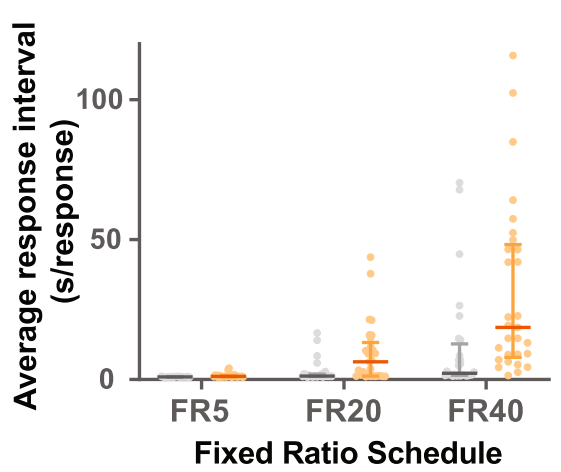

F

G
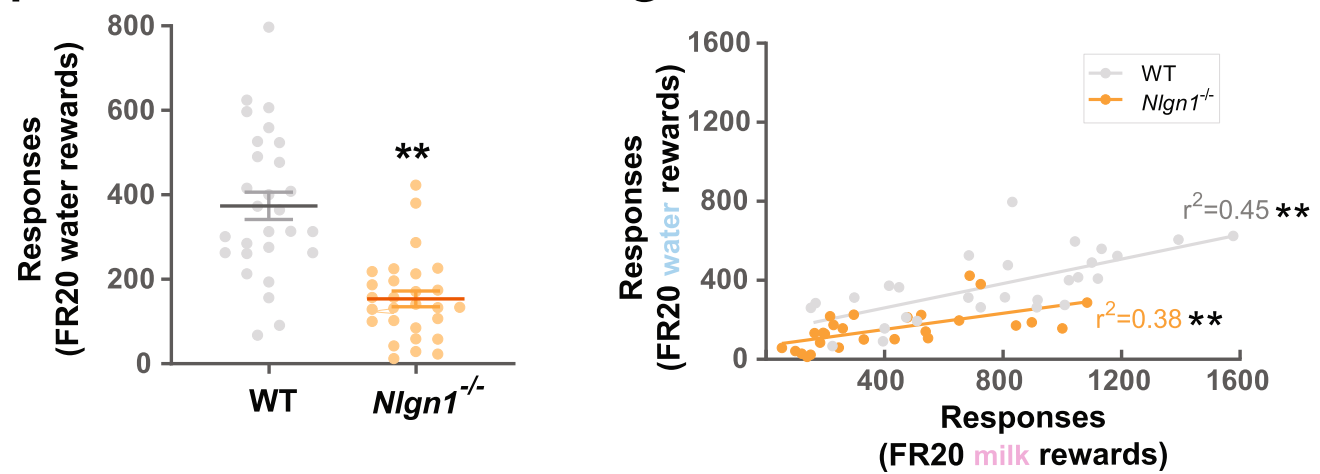

Fig. 4 (See legend on next page.) 


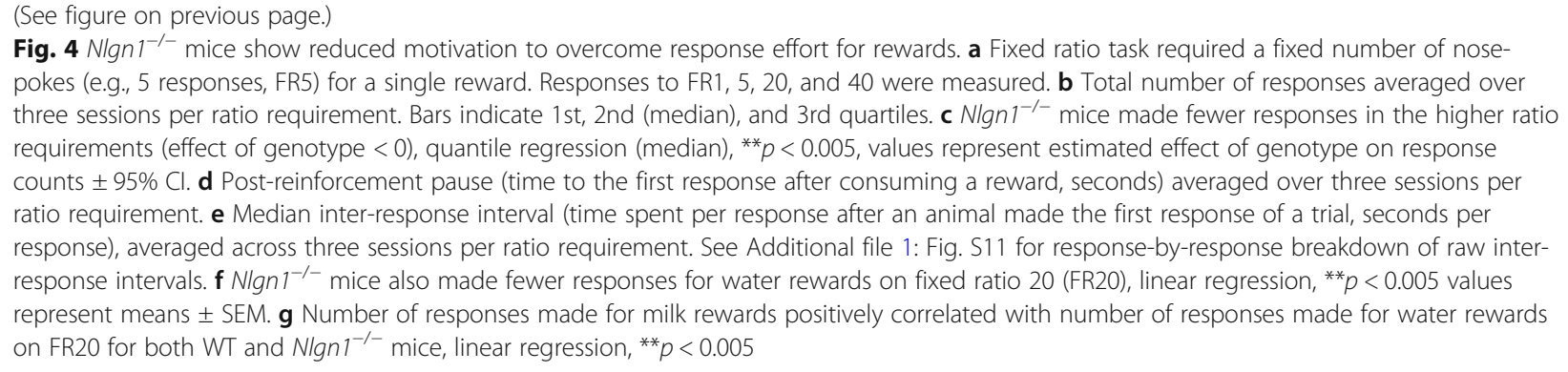

in the latencies to fall off an accelerating rotarod across repeated trials (Additional file 1: Fig. S15) highlighting loss of Nlgn1 does not impair intrinsic motor function; therefore, the observed changes in motivational measures are not due to locomotor capacity.

Motivation often refers to the ability to overcome physical effort to achieve a desirable outcome [46-49], but what if the desired outcome was to avoid punishment? We were therefore interested to know whether an increased aversion to physical effort to earn rewards would manifest as a decreased willingness to avoid punishment. To examine this, we next employed the Porsolt forced swim test (Fig. 5b) in which the choice to swim or "struggle" to escape the aversive situation of being immersed in water compared to immobility is taken to model behavioral despair [50-52]. Here, we surprisingly observed that $N \operatorname{lgn} 1^{-1-}$ mice spent significantly more time mobile than WT controls (Fig. 5f). These data suggest that the behavioral phenotype of $\mathrm{Nlgn}^{-/-}$mice cannot be described simply as a general reduction in the willingness to overcome effort cost.

\section{Convergence on a model of increased weighting on negative utilities}

Theoretical accounts for the potential cognitive mechanisms underlying decreased motivation have been reported $[44,53,54]$. We therefore considered the possibility that the seemingly opposing motivational phenotypes observed in reward- and punishment-driven contexts many converge on the same underlying mechanism. On reflection, in the Porsolt swim test both the fear of drowning and the effort of swimming incurs negative utilities. Therefore, Nlgn1 may not only be involved in estimating the cost of physical effort, but rather more broadly important for regulating the sensitivity to domain-general negative utilities (any undesirable consequences of actions/inaction). To explore this, we sought a theoretical model to capture the key behavioral observations of $N \operatorname{lgn} 1^{-1-}$ mice across tasks including (1) normal binary effort-matched choices between a correct and incorrect response in learning tasks (e.g., visual discrimination, object-location paired associate, reversal), (2) fewer responses emitted on a sequential fixed ratio task (e.g., FR5-40), and (3) higher mobility when immersed in water in the Porsolt swim test. We visualized the behavioral predictions of the theory by simulating the behavior of a simple reinforcement learning model using the three tasks mentioned, allowing us to describe precisely and unambiguously the assumptions, architecture, and predictions of the theory. We compared the simulated effect of changing the parameters in the theoretical model to the effect of Nlgn1 deletion in the experimental data.

In the model, the simulated agent selects actions by comparing the net utility of each available action, sum of positive and negative utilities weighted by two separate parameters (inspired by Collins and Frank [45], Fig. 6a). Importantly, these parameters affect only the weightings on learned action utilities but not the process of learning itself, allowing a potential dissociation between learning and action selection. We first considered how reducing the weighting on positive utilities $\left(\beta_{\mathrm{P}}\right)$ would impact the calculation of net utilities (Additional file 1: Fig. S16). Reducing the weighting on positive utilities promotes loweffort-low-reward actions (e.g., resting) leading to a reduction in responses made in the fixed ratio task, consistent with our observed experimental data in $N \operatorname{lgn} 1^{-/-}$mice. On the contrary, reducing the weighting on positive utilities renders the choice between correct and incorrect responding more random in the simulated binary choice task, because performance accuracy depends on the difference between the positive utilities associated with the correct and incorrect response. Here, we see this model does not capture the $N \operatorname{lgn} 1^{-/-}$phenotype in the pairwise visual discrimination, object-location paired associate learning, and reversal learning tasks.

Next, we considered how increasing the weighting on negative utilities $\left(\beta_{\mathrm{N}}\right)$ impacts calculation of net utilities (Fig. 6b). We found increasing $\beta_{\mathrm{N}}$ (i) did not alter the learning curves for binary choices because correct and incorrect responding required the same physical effort, (ii) reduced the number of responses in the simulated fixed ratio tasks because the potential rewards of responding were more heavily discounted by the physical effort incurred, and (iii) increased mobility in the Porsolt swim test because increasing $\beta_{\mathrm{N}}$ had a greater effect on 
A
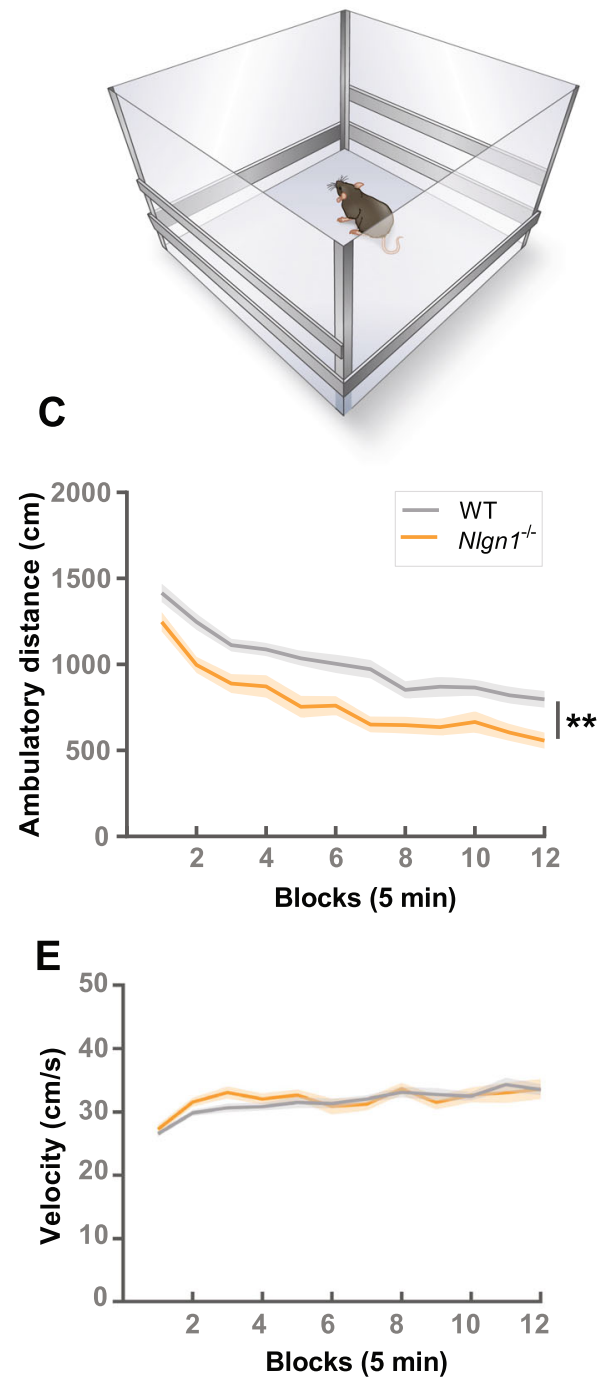

B

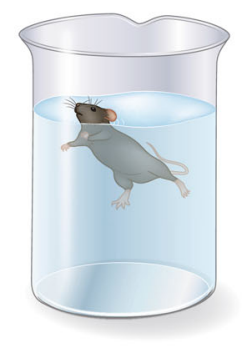

D
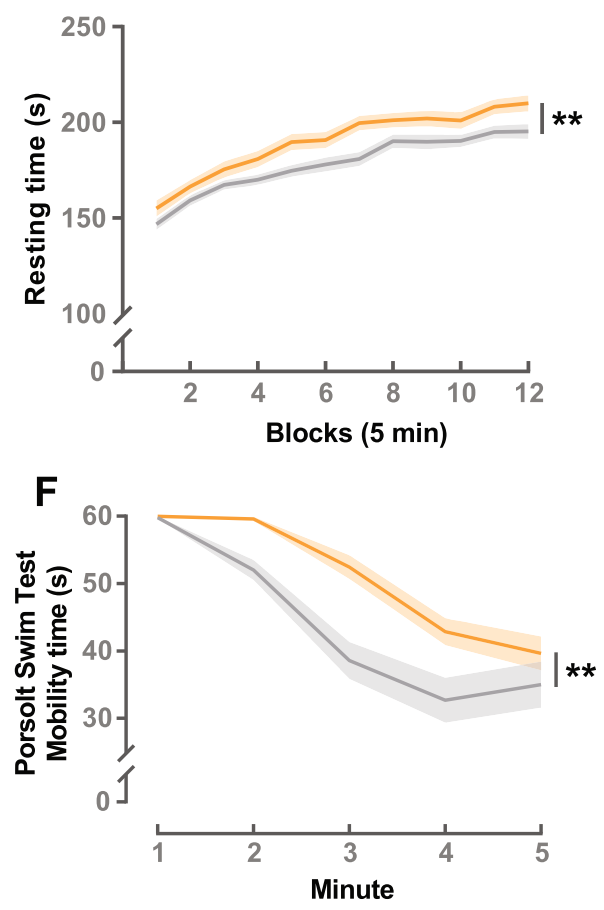

Fig. 5 Measuring motivational behavior outside an operant environment. a Open-field test. b Porsolt swim test. Nlgn $1^{-/-}$mice (with previous operant experience) show decreased exploration and spontaneous locomotor activity in a novel, open-field environment. c Ambulatory distance (centimeters) (generalized linear model ${ }^{* *} p<0.005$ ) and $\mathbf{d}$ resting time (seconds) (mixed-effects linear model $\left.{ }^{* *} p<0.005\right)$ but no changes in $\mathbf{e}$ velocity (centimeters/second) (mixed-effects linear model). Values represent means \pm SEM. f Nlgn $1^{-1-}$ mice showed increased mobility time (seconds) in the Porsolt swim test; two-way ANOVA, time bins collapsed for analysis, main effect of genotype ${ }^{* *} p<0.005$, values represent means \pm SEM

greater punishment, under the assumption that immersion in water has a greater negative utility than the physical effort of swimming. Together, an increased weighting on negative utilities can, at least in principle, capture the divergent phenotypes across reward- and punishment-driven tasks to suggest loss of Nlgn1 may be important for balancing the weighting on positive and negative utilities in reward-cost trade-off.

\section{Discussion}

Building on the extensive work on the molecular and signaling functions of Nlgn1 at the synapse, we investigated how the loss of Nlgn1 might impact components of decision-making. We found that Nlgn1 was not required for learning complex associative structures, or the subsequent updating of learned associations. However, Nlgn $1^{-1-}$ mice were consistently less motivated to overcome the effort cost to earn rewards across different reward-based free-operant tasks, but more motivated to exert effort to avoid an inescapable aversive situation. We suggest these divergent phenotypes converge on a model of increased weighting on negative utilities, highlighting a novel valence-dependent role of Nlgn1 in reward-cost trade-off. Our findings provide novel 


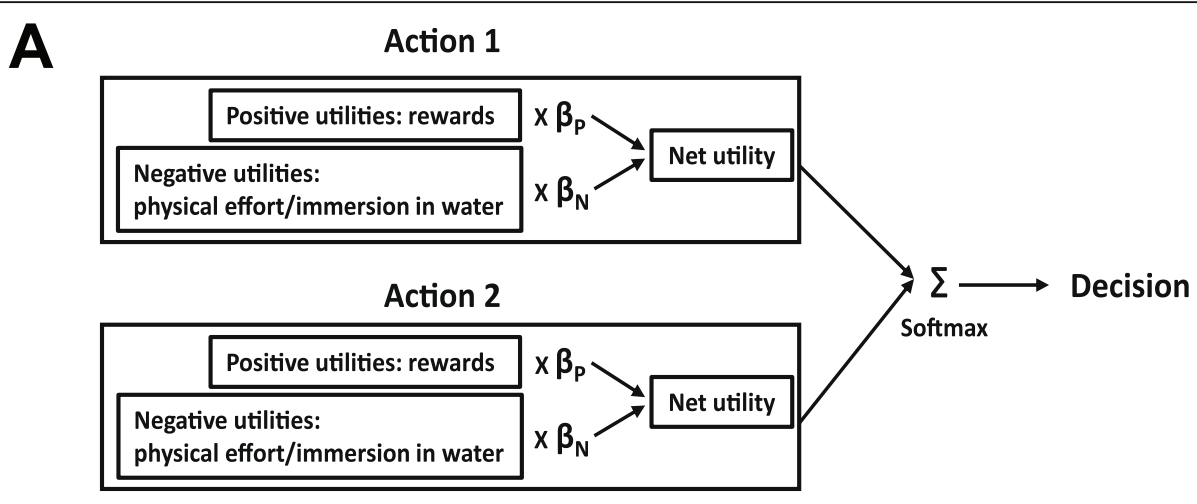

B

\section{Simulation}

Experiment

\section{$\beta_{\mathrm{N} \text { (negative utility) }}$}

1
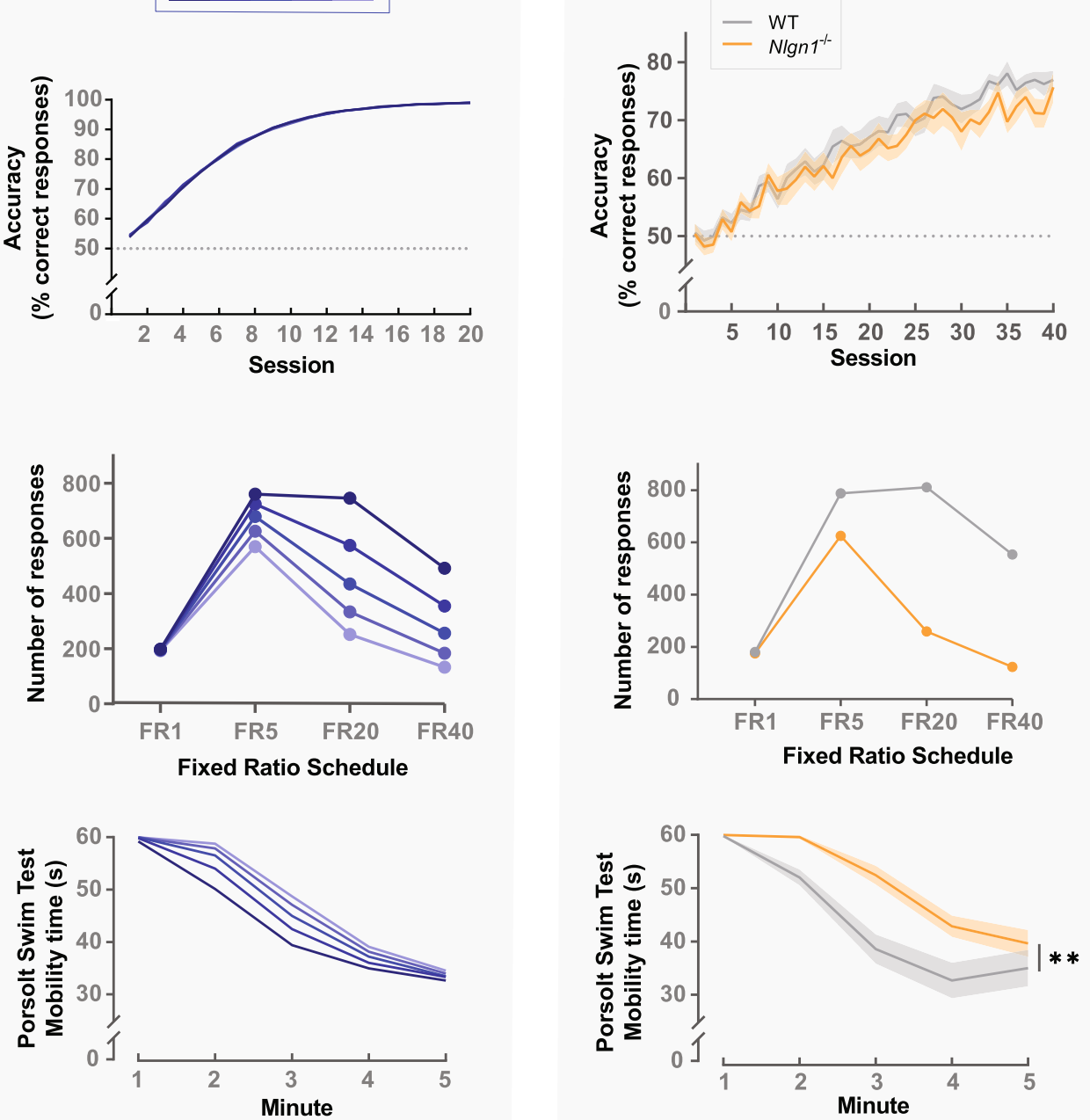

Fig. 6 (See legend on next page.) 
(See figure on previous page.)

Fig. $6 \mathrm{~A}$ model of increased weighting on negative utilities captures the observed NIgn $1^{-1-}$ behavioral phenotype across tasks. a An agent selects actions by comparing the net utility of each available action, sum of positive and negative utilities weighted by two separate parameters $\left(\beta_{p}\right.$ and $\beta_{N}$ ). The model assumes different types of positive and negative utilities are under the general control of $\beta_{p}$ and $\beta_{N}$ (e.g., $\beta_{N}$ affects both the weighting on physical effort as well as immersion in water where appropriate). Net utilities of potential actions are then fed into a softmax function for probabilistic action selection where actions with higher net utilities are selected with higher probabilities. $\mathbf{b}$ Left panel: Increasing $\beta_{\mathrm{N}}$ has (i) no impact on the choice between correct and incorrect responding in the simulated binary choice task, (ii) reduces the number of responses made in the simulated fixed ratio task where the choice is between responding (high-effort action) and resting (low-effort action), and (iii) increases mobility in the simulated Porsolt swim test where the choice is between swimming (high-effort action) and resting (low-effort action). b Right panel: For comparison, our experimental data from (i) object-location paired associate learning (PAL) task, (ii) fixed ratio task, and (iii) Porsolt swim test

evidence that updates current views to show Nlgn1 is essential for regulating distinct cognitive processes underlying decision-making. This work also demonstrates that learning and motivational processes can be dissociated.

It is widely held that NMDA receptor function and long-lasting forms of synaptic plasticity are required for various forms of learning (e.g., [28-31, 35, 55]). Based on previous findings that loss of Nlgn1 robustly impairs NMDA receptor function and synaptic plasticity in various brain regions (e.g., $[7,14,15,17-20,25,26])$ and decreases spatial learning and memory in the Morris water maze [14], we initially speculated that Nlgn1 loss of function would likely impair the ability to learn complex associations in our various touchscreen-based tasks. Indeed, disrupting NMDA receptor signaling and plasticity has been shown to impair performance in these touchscreen-based tasks employing training parameters similar to our study $[35,55-58]$. It is therefore striking that in our studies $N \operatorname{lgn} 1^{-1-}$ mice showed normal acquisition of complex associative structures and were able to modify learned associations. Towards collectively reconciling these findings, our data emphasize the capacity for synaptic signaling and plasticity does not map uniformly onto all forms of learning, thus highlighting the complexity in reducing behavioral processes to discrete cellular mechanisms. In line with this, Nlgn1 overexpression enhances NMDA receptor transmission without impairing LTP but nonetheless decreases learning and memory in the Morris water maze [24]. Given the many different levels of neural architecture between behavior and synaptic molecules, as well as the multiple components and interactions within each level, our findings caution interpretations between behavior and synaptic signaling and emphasize the relationship between the cellular mechanisms and the emergence of distinct forms of cognitive behavior is highly complex and indirect.

It is also worth noting that functional studies on synaptic proteins (including Nlgn1) have predominantly been performed in vitro and often limited to isolated brain regions (e.g., the hippocampus) and cell types (e.g., pyramidal neurons). Nlgn1 is predominantly expressed at excitatory synapses, but it has also been documented in oligodendrocytes [59] and astrocytes [60] and potentially plays a role in inhibitory synapse formation [61]; thus, unraveling cell-type-specific roles of neuroligins presents another layer of complexity (e.g., $[62,63])$. The synaptic mechanisms required for the emergence of large-scale neural representations during complex learning, and how these might be regulated by molecular components that structurally organize synapses therefore remains largely unknown. One way forward would be to examine network-level synaptic changes during behavior. Technological advances in recent years now allow in vivo monitoring of neural dynamics of large populations of cells across multiple brain regions in awakebehaving animals with high cellular specificity. These emerging approaches offer new opportunities for probing how synaptic mechanisms influence large-scale neural dynamics underlying complex behavior.

Despite an intact capacity to acquire and flexibly update learned information, we found that loss of Nlgn1 alters motivational processing, impacting reward-cost trade-off. Action selection generally requires weighing up both the expected positive and negative consequences of available actions. We see a reduced willingness to overcome response effort for reward in touchscreen-based tasks. Theoretical models have been proposed to address the dissociation between learning and motivation for rewards, potentially explaining our dissociation [44, 54]. According to these theories, an animal could be less motivated to overcome the effort cost of responding yet maintain the normal ability to choose between correct and incorrect responses due to either an underestimation of the average reward rate of the environment independent of any particular action, or an overestimation of the effort costs of potential actions. However, we additionally see an increased willingness to exert effort to escape an aversive situation in the Porsolt swim test. One interpretation may be that this is due to an exaggerated anxiety or fear response. However, there is no strong evidence to suggest that loss of Nlgn1 elevates response to aversive stimuli. Previous work has shown loss of Nlgn1 does not alter freezing behavior 
during contextual and cued fear conditioning [14], though knockdown of Nlgn1 in the amygdala of rats impairs the retention of fear memory [19]. $N \operatorname{lgn} 1^{-1-}$ mice also display normal behavior on various anxiety-related assays [14].

The divergent phenotypes observed in the rewardassociated touchscreen tasks and the punishmentassociated Porsolt forced swim test could be due to either changes in distinct or shared underlying mechanisms involved in decision-making processes. Our behavioral simulation data show that the seemingly opposing phenotypes could, in principle, converge on an increased weighting on negative utilities. Two key questions arise from this interpretation. First, is it plausible to assume a common currency for effort cost and other forms of punishment? Conceptually, this seems reasonable since physical cost, like other forms of punishment, is a decision variable which animals should seek to minimize all else being equal [64]. Empirically, functional magnetic resonance imaging (fMRI) data suggest that hemodynamic responses in select brain regions such as the anterior insula cortex correlate with both physical cost and other forms of punishment [64-68]. Second, is it plausible that the brain affords dissociable neural computations and hardware for reward and punishment? It is generally accepted that there is at least partial dissociation between the neural implementation of reward and punishment [69-71]. Notwithstanding the lack of a consensus, reward and punishment processing has been shown to be differentially implemented by activity [72] and identity of distinct subsets of dopaminergic neurons [73-75] or opponent dopaminergic and serotoninergic signaling $[54,76]$.

We acknowledge that our proposed model of increased weighting on negative utilities is, at this point, tentative and requires further investigations. Indeed, our intention was to additionally assess whether $\mathrm{Nlgn}^{-1-}$ mice would exert more effort to avoid punishment in an operant paradigm comparable to our touchscreen tasks. To address this, we attempted to train mice to press a lever to either avoid an upcoming footshock or to terminate it. Unfortunately, C57BL/6 mice were unable to acquire the action-safety contingency in both task variants. It appears that in the face of an aversive outcome, mice can escape but struggle to acquire trained actions to prevent it. This phenotype was also recently reported in rats [77] showing that while rats froze in response to footshock and the conditioned cue, they did not robustly detect the contingency between lever-press and footshock. Future studies on positive/negative utility tradeoff will benefit from the development of robust aversive instrumental paradigms for rats and mice.

Behavior is complex; thus, it is not surprising that a single behavioral assay cannot often reliably assess the cognitive construct in question. Our data, cultivated from detailed analyses across a battery of tests, highlight the value of a deep behavioral dissection that enables the synthesis of a cross-task interpretation from multiple paradigms. These touchscreen tests provide a unique opportunity to obtain such a comprehensive dataset consisting of multiple behavioral measures that are unique and shared across tests, assessed within the same testing environment in a controlled and comparable manner. Extending previous work using these touchscreen operant paradigms, we also modified our data analysis approach to employ trial-by-trial level analyses with regression models. This approach is tailored to describing complex behavioral data sampled at the trial level and across multiple sessions, yet it is under-utilized in rodent behavioral studies where session or stage-level summary measures are commonly calculated, potentially missing valuable depth in data. Exploiting this trial-level approach, we incorporated various latencies into the analysis of free-operant behavior which more accurately represents the paradigm as a continuous decision process rather than discrete trials. We also show, for the first time, that we can further dissect response latencies to sub-components that differentially contribute to decision-making and learning (e.g., stimulus-selection vs stimulus-approach latency). Measuring latencies for different response epochs during behavioral responding within the touchscreen chambers is a powerful parameter, analogous to human cognitive measures such as processing speed and reaction times, which have been difficult to be capture in other rodent behavioral assays.

Finally, human mutations in $N L G N$ genes, including NLGN1, have been reported in neurodevelopmental disorders including autism spectrum disorder $[78,79]$. It is noteworthy that depression, affective, and anxiety symptoms are highly comorbid with neurodevelopmental disorders, supporting the need to dissect distinct components of cognitive behavior and gain a deeper understanding of the transdiagnostic psychological processes that can be effectively used as behavioral markers of disease.

\section{Conclusions}

Our work updates canonical views of Nlgn1, a key postsynaptic cell-adhesion molecule, in cognitive behavior to show its critical for tuning valence-dependent processes regulating motivation, but not learning and updating complex associative structures. Our observations emphasize the relationship between the cellular mechanisms that support the emergence of distinct forms of cognitive behavior is highly complex and indirect; thus, the need to scrutinize established interpretations that impaired synaptic transmission/plasticity necessarily and uniquely lead to learning deficits. We highlight the value 
and advantage of our detailed behavioral dissection, exploiting a battery of free-operant touchscreen-based tests that enables the synthesis of a cohesive behavioral interpretation by identifying cross-task phenotypes. Extending this, we showcase response latencies can be dissected to examine their differential contribution to decision-making and learning, providing avenues for capturing different response epochs during behavioral responding analogous to human cognitive measures such as processing speed and reaction times that are not accessible in most rodent behavioral assays. We hope our analyses and approaches provide useful tools as the neuroscience community expand the integration of in vivo recordings and imaging during complex cognitive behavior in systems neuroscience. We demonstrate that learning and motivational processes can be dissociated in an animal model, providing insights into how human mutations in synapse genes that are expressed throughout the brain can selectively impact specific cognitive constructs, thus manifesting as disruptions in distinct symptoms. Of importance, depression, affective, and anxiety symptoms are highly comorbid with neurodevelopmental disorders. Our work provides evidence of being able to dissect distinct components of cognitive behavior in preclinical animal models, towards having robust models that enable deeper understandings into transdiagnostic behavioral markers of disease.

\section{Methods}

\section{Animals and housing}

Heterozygous $\mathrm{NlgnI}^{+/-}$mice were obtained from Prof. Nils Brose, generated by homologous recombination of embryonic stem cells deleting exon sequences covering the translational start site and $546 \mathrm{bp}$ of $5^{\prime}$ coding sequence of the murine Nlgn1 gene [80], and backcrossed more than 10 generations on a C57BL/6 background. $N \operatorname{lgn} 1^{-1-}$ mice and WT littermate matched controls were generated at The Florey by mating heterozygous females and males. Mice were weaned at 3-4 weeks of age and housed in mixed genotype groups of 2-4 per cage with food and water available ad libitum. Bedding consisted of sawdust chips $2 \mathrm{~cm}$ deep and tissue paper for nesting material. At $\sim 10$ weeks of age, mice were moved from individually ventilated cages to open-top cages in a humidity and temperature-controlled holding room maintained on a 12:12-h reversed light/dark cycle (lights off at 07:00). Mice were acclimatized to these conditions for a minimum of 1 week prior to handing. Pre-training began at $\sim 12$ weeks of age. All behavioral testing was conducted during the dark active phase of the cycle, with the experimenter blinded to genotype during behavioral testing. All procedures were approved by The Florey Institute of Neuroscience and Mental Health Animal Ethics Committee.

\section{Cohorts of mice used for behavioral testing}

A total of 6 cohorts of mice were used in the present study (see Additional file 1: Fig. S1 for a schematic of sequence of tasks for each cohort). Cohort 1 (WT: $n=12$ female/ $n=15$ male; $N \lg n 1^{-1-}: n=13$ female $/ n=13$ male) was tested in the pairwise visual discrimination, reversal learning, object-location paired associate learning, and extinction learning tasks. When a single cohort of animals was tested on multiple touchscreen-based tasks, mice were placed back on free-feeding for $\sim 2$ weeks and baseline weights updated prior to commencing food restriction for the next task. Cohort 2 (WT: $n=14$ female $/ n=14$ male; $N \operatorname{lgn} 1^{-1-}: n=14$ female $/ n=17$ male) was tested in the fixed ratio task (FR1-40) with strawberry milk rewards and fixed ratio 20 (FR20) task with water rewards. Cohort 3 (WT: $n=11$ female $/ n=12$ male; $N \lg n 1^{-/}: n=7$ female/ $n=12$ male) and cohort 4 (WT: $n=6$ female $/ n=4$ male; Nlgn $^{-1-}: n=6$ female/ $n=4$ male) were tested in the progressive ratio task, spontaneous locomotor activity, and accelerating rotarod tests. Cohort 5 (WT: $n=13$ female/ $n=10$ male; $\operatorname{Nlgn}^{-1-}: n=10$ female $/ n=12$ male) was tested in the Porsolt forced swim test following $\sim 2$ weeks simple operant training for a different study not included in this paper. Cohort 6 (WT: $n=13$ female $/ n=16$ male; $N \lg n 1^{-/-}: n=11$ female/ $n=16$ male) was experimentally naive and tested for spontaneous locomotor activity. For all non-touchscreen-based tasks, mice were not food restricted when tested.

\section{Rodent touchscreen operant tasks Apparatus}

Touchscreen testing was conducted in the BusseySaksida mouse touchscreen operant system (Campden Instruments Ltd., UK). Stimulus presentation, task parameters, and data recording were controlled through Whisker Server and ABET II Touch software (Campden Instruments Ltd., UK). The two-hole mask was used for the pairwise visual discrimination and reversal learning tasks, and the three-whole mask used for the objectlocation paired associate learning, extinction learning, fixed ratio, and progressive ratio tasks.

\section{Touchscreen pre-training}

Pre-training and food restriction were conducted as previously described [36, 37, 42]. Before testing, mice were first food restricted to $85-90 \%$ free-feeding body weight. Mice were then trained through five phases for instrumental conditioning to learn to selectively nose-poke stimuli displayed on the touchscreen in order to obtain a liquid reward (strawberry milk, Devondale, Australia; $20 \mu \mathrm{l}$ rewards for all touchscreen tests). All animals received one daily session for all touchscreen testing. Mice were required to reach a set performance criterion for each phase before advancing to the next phase. Briefly, 
mice were habituated (phase 1, Habituation) to the touchscreen chamber and to consuming liquid rewards from the reward magazine or receptacle for two 30-min sessions (criterion $=$ consume $200 \mu \mathrm{l}$ of liquid reward freely available in the reward receptacle at each session). For phases 2-5, a trial did not advance until the reward was consumed. In phase 2 (Initial Touch) or the Pavlovian stage, a single visual stimulus was displayed on the screen for $30 \mathrm{~s}$, after which the disappearance of the stimulus coincided with delivery of a reward $(20 \mu \mathrm{l})$, presentation of a tone and illumination of the reward receptacle (criterion $=30$ trials within $60 \mathrm{~min}$ ). A nosepoke response to the stimulus during the 30 -s window was rewarded with 3 times the reward amount to encourage responding. In phase 3 (Must Touch), mice had to nose-poke visual stimuli displayed on the screen to obtain a reward (criterion $=30$ trials within $60 \mathrm{~min}$ ). Mice then learned to initiate a new trial with a head entry into the reward receptacle (phase 4, Must Initiate, criterion $=30$ trials within $60 \mathrm{~min}$ ). In phase 5 , responses at a blank part of the screen during stimulus presentation produced a 5-s timeout (signaled by illumination of the house light and no delivery of reward) to discourage indiscriminate responding (criterion $=21 / 30$ correct responses within $60 \mathrm{~min}$ on 2 consecutive days). If another response to a blank part of the screen during stimulus presentation was made, there was a 5-s inter-trial interval (ITI), and then the same trial was repeated (the same stimulus presented in the same screen location, termed a "correction trial") until the mouse made a correct response. Therefore, phases 2-5 consisted of 30 trials (pseudorandom first-presentation), and phase 5 also included an unlimited number of correction trials.

\section{Pairwise visual discrimination and reversal learning}

The pairwise visual discrimination (PD) and reversal learning (RL) tasks were conducted like that previously described [36, 37, 42]. Briefly, mice were trained to discriminate between two novel, equiluminescent visual stimuli (left and right diagonal stripes) displayed pseudorandomly across two locations with equal number of appearances at each location. Stimuli were $5 \mathrm{~cm} \times 5 \mathrm{~cm}$ in size separated by $3 \mathrm{~cm}$ between stimuli and displayed 2 $\mathrm{cm}$ from the bottom of the touchscreen and $\sim 5.5 \mathrm{~cm}$ away from the sides of the touchscreen. Response to one stimulus resulted in reward delivery $\left(\mathrm{S}_{+}\right.$, correct response), followed by a pseudorandom trial (maximum 30 per session); response to the other stimulus resulted in a 5-s timeout, illumination of the house light followed by a correction trial. The same stimulus configuration was presented on correction trials until a correct response was made and a reward was delivered. Correction trials were not counted towards the trial limit or percentage of correct responses of a session. The designation of $\mathrm{S}_{+}$and $\mathrm{S}-$ was counterbalanced within genotype and sex groups. Mice were trained to an acquisition criterion of $\geq 80 \%$ correct responses on two consecutive sessions. Following the acquisition of the visual discrimination task, mice were immediately moved on to the reversal leaning task, where the previously acquired reward contingencies were reversed. Reversal learning was assessed across 20 sessions.

\section{Object-location paired associate learning}

The object-location paired associate learning (PAL) task was conducted as previously described [36, 37]. Briefly, mice were trained to acquire reward associations jointly defined by visual stimuli (flower, plane, and spider) and their assigned correct spatial locations on the touchscreen (left, center, and right, respectively). Stimuli were 5 $\mathrm{cm} \times 5 \mathrm{~cm}$ in size separated by $2 \mathrm{~cm}$ between stimuli and displayed $2 \mathrm{~cm}$ from the bottom and $\sim 2.5 \mathrm{~cm}$ away from the sides of the touchscreen. For each trial, only two objects were presented: one object in its correct location $(\mathrm{S}+)$ and the other object in one of two incorrect locations (S-); therefore, there were six possible trial types. A nose-poke to the S+ resulted in delivery of a reward followed by a pseudorandom trial (maximum 36 per session), and incorrect responses resulted in a 5-s timeout followed by correction trial. Visuospatial learning in the PAL task was assessed across 40 sessions.

\section{Instrumental extinction learning}

The instrumental extinction learning task was conducted similar to that previously described [37, 42]. Mice were first trained to make a nose-poke response to a single white square displayed on the touchscreen (stimulus was $3 \mathrm{~cm} \times 3 \mathrm{~cm}$ in size, displayed $3 \mathrm{~cm}$ from the bottom and $\sim 10.5 \mathrm{~cm}$ away from the sides of the touchscreen) for a reward until reaching a set acquisition criterion (30 trials in $<12.5 \mathrm{~min}$ on five consecutive sessions). Following acquisition, instrumental extinction was assessed where responses were no longer rewarded (30 trials per session tested across 6 sessions). During extinction, the visual stimulus was displayed for $10 \mathrm{~s}$ on each trial and animals could either make a response or an omission.

\section{Progressive ratio}

Details on testing the touchscreen-based progressive ratio task have been described previously [81]. Briefly, mice had to make nose-poke responses to a single white square displayed on the touchscreen (stimulus was 4 $\mathrm{cm} \times 4 \mathrm{~cm}$ in size, displayed $1.5 \mathrm{~cm}$ from the bottom and $\sim 10 \mathrm{~cm}$ away from the sides of the touchscreen) for a reward. Naive mice first underwent phases 1 and 2 of touchscreen pre-training, followed by one session each of fixed ratio (FR) schedules of 1 (FR1), FR2, and FR3 and three sessions of FR5 training where a fixed number 
of nose-pokes (1, 2, 3, and 5 respectively) were required for a reward. Mice were required to complete 30 trials in $60 \mathrm{~min}$ in each of the FR sessions (criterion). Once training criterion was reached, mice advanced to the progressive ratio stage where the number of nose-poke responses required to obtain a reward incremented by 4 after every trial $(1,5,9,13$, etc.) $)$ until animals reach a breakpoint. If no responses to the touchscreen or entries to the reward receptacle were detected for $5 \mathrm{~min}$, the session ended and the animal removed from the chamber. Mice were tested on 6 progressive ratio sessions.

\section{Fixed ratios}

Touchscreen-based fixed ratio testing was similar to that described for progressive ratio (mice had to make nosepoke responses to a single white square displayed on the touchscreen for a reward; stimulus was $4 \mathrm{~cm} \times 4 \mathrm{~cm}$ in size, displayed $1.5 \mathrm{~cm}$ from the bottom and $\sim 10 \mathrm{~cm}$ away from the sides of the touchscreen). Naive mice first underwent phases 1 and 2 of touchscreen pre-training followed by three sessions of FR1 and had to complete 30 trials within a 60-min session before advancing. During the next serial FR test stage, mice were given $60 \mathrm{~min}$ per session to make as many responses as they were willing to, and sessions did not terminate due to inactivity. Mice were tested on three sessions of FR1, FR5, FR20, and FR40 sequentially.

\section{Fixed ratio with water rewards}

Following the serial FR testing, mice were water-restricted with access to water limited to $1 \mathrm{~h}$ per day. Waterrestricted body weights were maintained between 85 and $90 \%$ of free-feeding body weight. Mice were tested on a FR20 schedule where 20 nose-poke responses were required to deliver a water reward $(20 \mu \mathrm{l})$ for three sessions. After each session, mice were returned to home cage and given 1-h free access to water.

\section{Touchscreen latency measures}

Across all our touchscreen tests, we assessed 4 latency measures (see Fig. 3a, Additional file 1: Fig. S4B). Initiation latency measures the time from the end of the inter-trial interval to trial initiation by head entry into the reward receptacle to commence a trial. Head entry triggers the presentation of stimuli. Stimulus-approach latency measures the time from exiting the reward receptacle to arriving in front of the touchscreen (breaking the front IR beam). Stimulus-selection latency measures the time from arriving in front of the touchscreen to nose-poking one of the stimuli on the touchscreen. Lastly, reward collection latency measures the time from delivery of the reward tone to head entry into the reward receptacle.

\section{Non-operant behavioral tests Spontaneous locomotor activity}

Mice were assessed for spontaneous locomotor activity in a novel open-field arena $(27.31 \mathrm{~cm}(\mathrm{~L}) \times 27.31 \mathrm{~cm}$ $(\mathrm{W}) \times 20.32 \mathrm{~cm}(\mathrm{H})$, Med Associates, St. Albans, VT, USA) using the Activity Monitor system and software (Med Associates, St. Albans, VT, USA). Animals were tested in darkness (to promote exploration) for $60 \mathrm{~min}$ to provide an adequate time window to capture the habituation of locomotor activity to a plateau level.

\section{Accelerating rotarod}

For motor coordination and learning on the accelerating rotarod, mice were exposed to three 5 -min trials across 3 consecutive days ( 9 trials in total). Mice were placed on a rotating rod (Ugo Basile, Gemonio, VA, Italy) facing forward (against the rotating direction of the rod) before acceleration started. Subsequently, the speed of the rotating rod accelerated from 4 to $40 \mathrm{rpm}$ and latency to fall off was manually recorded. Falls before the acceleration started were not recorded as failures. Passively rotating by clinging onto the rod was recorded as falls. Testing was conducted under low lighting settings (20 lx red light).

\section{Porsolt forced swim test}

Mice were individually placed into a beaker $(13 \mathrm{~cm}$ diameter) with $1.6 \mathrm{~L}$ of water $\left(23-25^{\circ} \mathrm{C}\right)$ for a single 5 -min session under ambient lighting (20-25 lx white light). Each session was video-recorded, and total mobility time throughout the 5-min session was measured (no time bins excluded). Scoring was obtained using the automated ForcedSwimScan software (CleverSys Inc., VA, USA) under previously optimized settings [82] eliminating the need for manual observer scoring.

\section{Data analysis}

Multi-session touchscreen choice data were analyzed with generalized (logistic) linear mixed models. This is motivated by (1) trial-by-trial binary nature of the data, (2) the need to estimate learning rates per time unit (session/trial), and (3) the non-linearity of learning curves. Touchscreen latency data across sessions were analyzed with quantile regressions to assess distribution-wide differences.

Effect size of task variables (stimulus location, session, correction trial, etc.), biological variables (genotype and sex), and interactions between a subset of variables (genotype $\times$ sex, genotype $\times$ session, etc.) on behavioral measures (accuracy, latencies, etc.) were estimated together with 95\% confidence intervals (CI) and statistical significance using various two-level mixed-effect general, generalized linear models or quantile regression (StataCorp, TX, USA). Mice were treated as level 2 clusters 
and random intercepts. Binary performance measures (correct/incorrect response, response/omission) were analyzed trial-by-trial using the generalized linear latent and mixed models (GLLAMM) program [38] with a logit link function, whereby the effects of task variables were expressed as odds ratios with an odds ratio of 1 indicating no effect (e.g., an effect of session $>1$ indicates response accuracy improves over sessions). Latency data were analyzed using quantile regressions with robust and clustered standard errors [83] from the 0.05 to 0.95 quantile at 0.05 steps to allow distribution-wide comparisons (see Additional file 1: Fig. S6), whereby effects of task variables were expressed as latency difference with 0 indicating no effect (e.g., an effect of genotype $>0$ for a given quantile indicates $N \operatorname{lgn} 1^{-1-}$ mice have longer latencies).

For spontaneous locomotor activity, ambulatory distance was analyzed with GLLAMM with a log link. Other performance measures were analyzed using a mixedeffects linear model if the performance measures were normally distributed or median regressions otherwise [83].

To analyze the effect of correction trials and reoccurring pseudorandom trials on accuracy, two additional binary variables were included in the models indicating whether a trial is a correction trial/reoccurring trial (correction trials were excluded in estimating the effect of reoccurring pseudorandom trials). Heteroskedasticityrobust standard errors adjusted for clustering within animals were used for all analyses.

\section{Behavior simulation \\ Agent}

A simple reinforcement learning agent learned the utility of an action following the classic Rescorla-Wagner rule [84]:

$$
Q_{t+1}(A)=Q_{t}(A)+\alpha \cdot\left[r_{t}-Q_{t}(A)\right]
$$

Here, $Q_{\mathrm{t}}(A)$ is the learned utility of a given action $A$ on trial $t, \alpha$ is the learning rate, and $r_{t}$ is the reinforcement received on trial $t$. Actions can have both positive and negative utilities (e.g., responding may result in rewards but also incurs effort). The net utility of a given action is given by the linear combination of its positive and negative utilities, the relative importance of which is controlled independently by $\beta_{P}$ and $\beta_{N}$ respectively:

$$
U(A)=\sum_{i}\left[I \cdot \beta_{P}+(1-I) \cdot \beta_{N}\right] \cdot Q_{i}(A)
$$

Here, $U(A)$ is the net utility of action $A, Q_{i}(A)$ is the different positive or negative utilities of $A, I$ is the indicator function:

$$
I=\mathbb{1}_{Q\left(A_{i}\right) \geq 0}\left(Q\left(A_{i}\right)\right)
$$

Such that,

$$
I=\left\{\begin{array}{l}
1 \text { if } Q\left(A_{i}\right) \geq 0 \\
0 \text { if } Q\left(A_{i}\right)<0
\end{array}\right.
$$

Action selection is given by a softmax function on the net utilities of potential actions

$$
P(A)=\frac{e^{U(A)}}{\sum_{i} e^{U\left(A_{i}\right)}}
$$

Here, $P(A)$ is the probability of choosing action $A$, which depends on the net utility of $A$ compared to that of alternative actions. For all our simulations, a choice is made between only two actions.

\section{Simulations}

Binary choice (two-armed bandit) task The reinforcement learning agent learned to choose between a correct and an incorrect response for 30 trials per session across 20 sessions. The correct response was always rewarded, and the incorrect response never rewarded. Both correct and incorrect responding incurred a negative utility of -1 representing the physical effort of responding.

Serial fixed ratio task The agent was trained sequentially through FR1, 5, 20, and 40 for three sessions on each ratio requirement where it chose between responding or resting. Responding resulted in a reward if the ratio requirement was met (positive utility) and incurred a negative utility of -1 representing the physical effort. Resting results in no reward but incurs a much smaller effort-related negative utility of -0.2 . Note that the designation of the alternative action as resting is arbitrary. The general idea is that an animal chooses between responding and other low-reward-low-effort actions. Time elapsed as the agent chose to either respond or rest, the session ended after 2700 timesteps roughly corresponding to a 2700 -s or 45 -min session.

Porsolt swim test The forced swim test was simulated as a choice between swimming and resting. Swimming was initialized with a utility of 0 representing that the agent initially believed that swimming will lead to a neutral outcome, and a utility of -1 represents the effort of swimming. Resting had a large negative utility of -10 representing the possibility of drowning but incurred no effort. Every time the agent chose to swim, it received a reinforcement of -9 thereby gradually learned by Eq. (1) that swimming did not markedly improve the situation therefore reduced mobility over time. For simplicity, the agent made 300 decisions over 300 timesteps roughly corresponding to a 5 -min session. 
Note the logic of the proposed model does not depend on the specific values of task parameters used for the simulations, which were chosen so that the behavioral simulations are quantitatively similar to the experimental data.

\section{Supplementary information}

Supplementary information accompanies this paper at https://doi.org/10. 1186/s12915-020-00848-7.

Additional file 1: Fig. S1. Sequence of tasks administered for different cohorts of $\mathrm{Nlgn}^{-1-}$ and wildtype mice in this study. Fig. S2. $\mathrm{Nlgn}^{-1-}$ and wildtype mice required similar number of sessions to acquire touchscreen pre-training. Fig. S3. Instrumental extinction learning curves. Fig. S4. Task dynamics and latency measures of the pairwise visual discrimination, reversal learning and object-location paired associate learning tasks. Fig. S5. Performance accuracy on trials across learning the pairwise visual discrimination, reversal learning and object-location paired associate learning tasks. Fig. S6. Latency data are highly skewed and poorly captured by a single summary measure. Fig. S7. Stimulusselection latency positively predicts response accuracy but not stimulusapproach latency. Fig. S8. Latency learning curves. Fig. S9. Number of responses made in fixed ratio task. Fig. S10. Post-reinforcement pause and average response interval in fixed ratio task. Fig. S11. Response-byresponse comparison of inter-response intervals between $\mathrm{Nlgnt}^{-1-}$ and wildtype mice. Fig. S12. Average number of responses to breakpoint in progressive ratio task. Fig. S13. N/gn $1^{-1-}$ mice show decreased number of responses for water rewards. Fig. S14. Experimentally naive $\mathrm{Nlgn}^{-1-}$ mice show subtle changes in exploration and spontaneous locomotor activity in a novel, open-field environment. Fig. S15. N/gn $1^{-1-}$ mice displayed normal motor coordination and learning on the accelerating rotarod test. Fig. S16. Simulated effect of decreasing the weighting on positive utilities $\left(\beta_{p}\right)$ in the calculation of net utilities.

Additional file 2: Table S1. Variables included in regression models.

\section{Abbreviations}

AMPA: a-Amino-3-hydroxyl-5-methyl-4-isoxazole-propionate; FR: Fixed ratio; GLLAMM: Generalized linear latent and mixed models; LTP: Long-term potentiation; Nlgn1: Neuroligin-1; NIgn $1^{-1-}$ : Neuroligin-1 knockout; NMDA: NMethyl-D-aspartate; PAL: Object-location paired associate learning; PD: Pairwise visual discrimination; PR: Progressive ratio; PSD: Postsynaptic density; RL: Reversal learning; WT: Wildtype

\section{Acknowledgements}

We thank Prof. Nils Brose (Max Planck Institute for Experimental Medicine, Göttingen Germany) for providing breeding founders of $\mathrm{Nlgn}^{+/-}$mice; Prof. Leonid Churilov for statistical advice; Shana Schokman and Clara Lee for genotyping; Brittany Cuic, Leah Payne, and Florey Core Animal Services for mouse husbandry; Jeremy Metha for programming assistance in attempts to develop an instrumental active avoidance task; and Debbie Maizels for manuscript artwork contribution.

\section{Authors' contributions}

J.L. and J.N. conceived and designed the experiments. J.L performed all experiments and analyses with assistance from J.T. in testing spontaneous locomotor activity and Porsolt swim test. J.L. and J.N. wrote the manuscript. The authors declare no competing interests. The authors read and approved the final manuscript.

\section{Funding}

This work was supported by an Australian Research Council Future Fellowship (FT140101327) and Brain and Behavior Research Foundation (NARSAD) Young Investigator Award to J.N., Australian Postgraduate Research Training Award to J.L, a National Health and Medical Research Council Project Grant (APP1083334), and philanthropic support from One In Five Mental Health Charity.

\section{Availability of data and materials}

Data that support the findings of this study have been made openly available using the Open Science Framework repository (https://osf.io/vfys8/, DOI: https://doi.org/10.17605/OSF.IO/VFYS8) and will also be available from MouseBytes (https://mousebytes.ca/home) [85]. Preprint of this manuscript was made available on bioRxiv on 02 January 2020 [86].

Ethics approval and consent to participate

All animal experiments were performed with approval from The Florey Institute of Neuroscience and Mental Health Animal Ethics Committee (Parkville, Australia) in accordance with Australian National Health and Medical Research Guidelines on Ethics in Animal Experiments.

\section{Consent for publication}

Not applicable.

\section{Competing interests}

The authors declare no competing interests.

Received: 14 April 2020 Accepted: 14 August 2020

Published online: 14 September 2020

\section{References}

1. Ichtchenko K, et al. Neuroligin 1: a splice site-specific ligand for betaneurexins. Cell. 1995;81(3):435-43.

2. Song JY, Ichtchenko K, Sudhof TC, Brose N. Neuroligin 1 is a postsynaptic cell-adhesion molecule of excitatory synapses. Proc Natl Acad Sci U S A. 1999;96(3):1100-5.

3. Irie M, et al. Binding of neuroligins to PSD-95. Science. 1997;277(5331):1511-5.

4. lida J, Hirabayashi S, Sato Y, Hata Y. Synaptic scaffolding molecule is involved in the synaptic clustering of neuroligin. Mol Cell Neurosci. 2004; 27(4):497-508.

5. Haas KT, et al. Pre-post synaptic alignment through neuroligin-1 tunes synaptic transmission efficiency. Elife. 2018;7:e31755.

6. Barrow SL, et al. Neuroligin1: a cell adhesion molecule that recruits PSD-95 and NMDA receptors by distinct mechanisms during synaptogenesis. Neural Dev. 2009:4:17.

7. Budreck EC, et al. Neuroligin-1 controls synaptic abundance of NMDA-type glutamate receptors through extracellular coupling. Proc Natl Acad Sci U S A. 2013;110(2):725-30

8. Heine $M$, et al. Activity-independent and subunit-specific recruitment of functional AMPA receptors at neurexin/neuroligin contacts. Proc Natl Acad Sci U S A. 2008;105(52):20947-52.

9. Mondin $\mathrm{M}$, et al. Neurexin-neuroligin adhesions capture surface-diffusing AMPA receptors through PSD-95 scaffolds. J Neurosci. 2011;31(38):13500-15.

10. Castellano C, Cestari V, Ciamei A. NMDA receptors and learning and memory processes. Curr Drug Targets. 2001;2(3):273-83.

11. Li F, Tsien JZ. Memory and the NMDA receptors. N Engl J Med. 2009;361(3): 302-3.

12. Lynch MA. Long-term potentiation and memory. Physiol Rev. 2004;84(1):87136.

13. Nicoll RA. A brief history of long-term potentiation. Neuron. 2017:93(2):281-90.

14. Blundell J, et al. Neuroligin-1 deletion results in impaired spatial memory and increased repetitive behavior. J Neurosci. 2010;30(6):2115-29.

15. Chubykin AA, et al. Activity-dependent validation of excitatory versus inhibitory synapses by neuroligin-1 versus neuroligin-2. Neuron. 2007;54(6): 919-31.

16. Espinosa F, Xuan Z, Liu S, Powell CM. Neuroligin 1 modulates striatal glutamatergic neurotransmission in a pathway and NMDAR subunit-specific manner. Front Synaptic Neurosci. 2015;7:11.

17. Jiang $\mathrm{M}$, et al. Conditional ablation of neuroligin-1 in CA1 pyramidal neurons blocks LTP by a cell-autonomous NMDA receptor-independent mechanism. Mol Psychiatry. 2017;22(3):375-83.

18. Jung SY, et al. Input-specific synaptic plasticity in the amygdala is regulated by neuroligin-1 via postsynaptic NMDA receptors. Proc Natl Acad Sci U S A. 2010:107(10):4710-5

19. Kim J, et al. Neuroligin-1 is required for normal expression of LTP and associative fear memory in the amygdala of adult animals. Proc Natl Acad Sci U S A. 2008;105(26):9087-92.

20. Wu XT, et al. Neuroligin-1 signaling controls LTP and NMDA receptors by distinct molecular pathways. Neuron. 2019;102(3):621. 
21. Nam Cl, Chen L. Postsynaptic assembly induced by neurexin-neuroligin interaction and neurotransmitter. Proc Natl Acad Sci U S A. 2005;102(17): 6137-42.

22. Futai $\mathrm{K}$, et al. Retrograde modulation of presynaptic release probability through signaling mediated by PSD-95-neuroligin. Nat Neurosci. 2007;10(2):186-95.

23. Kwon $\mathrm{HB}$, et al. Neuroligin-1-dependent competition regulates cortical synaptogenesis and synapse number. Nat Neurosci. 2012;15(12):1667-74.

24. Hoy $J$, et al. Neuroligin1 drives synaptic and behavioral maturation through intracellular interactions. J Neurosci. 2013;33(22):9364-84.

25. Jedlicka $P$, et al. Neuroligin-1 regulates excitatory synaptic transmission, LTP and EPSP-spike coupling in the dentate gyrus in vivo. Brain Struct Funct. 2015;220(1):47-58.

26. Shipman SL, Nicoll RA. A subtype-specific function for the extracellular domain of neuroligin 1 in hippocampal LTP. Neuron. 2012;76(2):309-16.

27. Dahlhaus $R$, et al. Overexpression of the cell adhesion protein neuroligin-1 induces learning deficits and impairs synaptic plasticity by altering the ratio of excitation to inhibition in the hippocampus. Hippocampus. 2010;20(2): 305-22

28. Whitlock JR, Heynen AJ, Shuler MG, Bear MF. Learning induces long-term potentiation in the hippocampus. Science. 2006;313(5790):1093-7.

29. Rogan MT, Staubli UV, LeDoux JE. Fear conditioning induces associative long-term potentiation in the amygdala. Nature. 1997;390(6660):604-7.

30. Reynolds JN, Hyland BI, Wickens JR. A cellular mechanism of reward-related learning. Nature. 2001;413(6851):67-70.

31. Nabavi S, et al. Engineering a memory with LTD and LTP. Nature. 2014; 511(7509):348-52.

32. Moser El, Krobert KA, Moser MB, Morris RG. Impaired spatial learning after saturation of long-term potentiation. Science. 1998;281(5385):2038-42.

33. Morris RG, Anderson E, Lynch GS, Baudry M. Selective impairment of learning and blockade of long-term potentiation by an N-methyl-Daspartate receptor antagonist, AP5. Nature. 1986;319(6056):774-6.

34. Giese KP, Fedorov NB, Filipkowski RK, Silva AJ. Autophosphorylation at Thr286 of the alpha calcium-calmodulin kinase II in LTP and learning. Science. 1998;279(5352):870-3.

35. Brigman $J \mathrm{~L}$, et al. Impaired discrimination learning in mice lacking the NMDA receptor NR2A subunit. Learn Mem. 2008;15(2):50-4.

36. Horner $A E$, et al. The touchscreen operant platform for testing learning and memory in rats and mice. Nat Protoc. 2013:8(10):1961-84.

37. Nithianantharajah J, et al. Synaptic scaffold evolution generated components of vertebrate cognitive complexity. Nat Neurosci. 2013;16(1):16-24

38. Rabe-Hesketh S, Skrondal A, Pickles A. Maximum likelihood estimation of limited and discrete dependent variable models with nested random effects. J Econ. 2005;128(2):301-23.

39. Graybeal C, et al. Strains and stressors: an analysis of touchscreen learning in genetically diverse mouse strains. PLoS One. 2014;9(2):e87745.

40. Kalueff AV, et al. Neurobiology of rodent self-grooming and its value for translational neuroscience. Nat Rev Neurosci. 2016;17(1):45-59.

41. Crawley JN. Mouse behavioral assays relevant to the symptoms of autism. Brain Pathol. 2007:17(4):448-59.

42. Mar AC, et al. The touchscreen operant platform for assessing executive function in rats and mice. Nat Protoc. 2013;8(10):1985-2005.

43. Aberman JE, Salamone JD. Nucleus accumbens dopamine depletions make rats more sensitive to high ratio requirements but do not impair primary food reinforcement. Neuroscience. 1999;92(2):545-52.

44. Niv $Y$, Daw ND, Joel D, Dayan P. Tonic dopamine: opportunity costs and the control of response vigor. Psychopharmacology. 2007;191(3):507-20.

45. Collins AG, Frank MJ. Opponent actor learning (OpAL): modeling interactive effects of striatal dopamine on reinforcement learning and choice incentive. Psychol Rev. 2014;121(3):337-66.

46. Pessiglione M. Why don't you make an effort? Computational dissection of motivation disorders. Euro Psychiatry. 2014;29(8):541.

47. Pessiglione M, Vinckier F, Bouret S, Daunizeau J, Le Bouc R. Why not try harder? Computational approach to motivation deficits in neuro-psychiatric diseases. Brain. 2018:141(3):629-50.

48. Salamone JD, Correa M. The mysterious motivational functions of mesolimbic dopamine. Neuron. 2012;76(3):470-85.

49. Salamone JD, Yohn SE, Lopez-Cruz L, San Miguel N, Correa M. Activational and effort-related aspects of motivation: neural mechanisms and implications for psychopathology. Brain. 2016;139(Pt 5):1325-47.

50. Molendijk ML, de Kloet ER. Immobility in the forced swim test is adaptive and does not reflect depression. Psychoneuroendocrino. 2015;62:389-91.
51. West AP. Neurobehavioral studies of forced swimming - the role of learning and memory in the forced swim test. Prog Neuro-Psychoph. 1990;14(6):863-77.

52. de Kloet ER, Molendijk ML. Coping with the Forced Swim Stressor: Towards Understanding an Adaptive Mechanism. Neural Plast. 2016;2016:6503162.

53. McClure SM, Daw ND, Montague PR. A computational substrate for incentive salience. Trends Neurosci. 2003;26(8):423-8.

54. Dayan P. Instrumental vigour in punishment and reward. Eur J Neurosci. 2012;35(7):1152-68.

55. Brigman JL, et al. GluN2B in corticostriatal circuits governs choice learning and choice shifting. Nat Neurosci. 2013;16(8):1101-10.

56. Ryan TJ, et al. Evolution of GluN2A/B cytoplasmic domains diversified vertebrate synaptic plasticity and behavior. Nat Neurosci. 2013;16(1):25-32.

57. Talpos JC, Winters BD, Dias R, Saksida LM, Bussey TJ. A novel touchscreenautomated paired-associate learning (PAL) task sensitive to pharmacological manipulation of the hippocampus: a translational rodent model of cognitive impairments in neurodegenerative disease. Psychopharmacology. 2009:205(1):157-68.

58. Marquardt K, et al. Impaired cognitive flexibility following NMDAR-GluN2B deletion is associated with altered orbitofrontal-striatal function. Neuroscience. 2019;404:338-52.

59. Hughes AN, Appel B. Oligodendrocytes express synaptic proteins that modulate myelin sheath formation. Nat Commun. 2019;10(1):4125.

60. Stogsdill JA, et al. Astrocytic neuroligins control astrocyte morphogenesis and synaptogenesis. Nature. 2017;551(7679):192-7.

61. Xia QQ, et al. Neuroligins differentially mediate subtype-specific synapse formation in pyramidal neurons and interneurons. Neurosci Bull. 2019;35(3): 497-506.

62. Zhang B, Sudhof TC. Neuroligins are selectively essential for NMDAR signaling in cerebellar stellate interneurons. J Neurosci. 2016;36(35):9070-83.

63. Polepalli JS, et al. Modulation of excitation on parvalbumin interneurons by neuroligin-3 regulates the hippocampal network. Nat Neurosci. 2017;20(2):219-29.

64. Skvortsova V, Palminteri S, Pessiglione M. Learning to minimize efforts versus maximizing rewards: computational principles and neural correlates. Neurosci. 2014;34(47):15621-30.

65. Prevost C, Pessiglione M, Metereau E, Clery-Melin ML, Dreher JC. Separate valuation subsystems for delay and effort decision costs. J Neurosci. 2010; 30(42):14080-90

66. Samanez-Larkin GR, Hollon NG, Carstensen LL, Knutson B. Individual differences in insular sensitivity during loss anticipation predict avoidance learning. Psychol Sci. 2008;19(4):320-3.

67. Seymour B, et al. Opponent appetitive-aversive neural processes underlie predictive learning of pain relief. Nat Neurosci. 2005;8(9):1234-40.

68. Pessiglione M, Seymour B, Flandin G, Dolan RJ, Frith CD. Dopaminedependent prediction errors underpin reward-seeking behaviour in humans. Nature. 2006:442(7106):1042-5.

69. Seymour B, Maruyama M, De Martino B. When is a loss a loss? Excitatory and inhibitory processes in loss-related decision-making. Curr Opin Behav Sci. 2015;5:122-7.

70. Pessiglione M, Delgado MR. The good, the bad and the brain: neural correlates of appetitive and aversive values underlying decision making Curr Opin Behav Sci. 2015:5:78-84.

71. Tye KM. Neural circuit motifs in valence processing. Neuron. 2018;100(2):436-52.

72. Maia TV, Frank MJ. From reinforcement learning models to psychiatric and neurological disorders. Nat Neurosci. 2011;14(2):154-62.

73. Brooks AM, Berns GS. Aversive stimuli and loss in the mesocorticolimbic dopamine system. Trends Cogn Sci. 2013;17(6):281-6.

74. Matsumoto M, Hikosaka O. Two types of dopamine neuron distinctly convey positive and negative motivational signals. Nature. 2009;459(7248):837-41.

75. Hauser TU, Eldar E, Dolan RJ. Separate mesocortical and mesolimbic pathways encode effort and reward learning signals. Proc Natl Acad Sci U S A. 2017:114(35):E7395-404.

76. Daw ND, Kakade S, Dayan P. Opponent interactions between serotonin and dopamine. Neural Netw. 2002;15(4-6):603-16.

77. Jean-Richard-Dit-Bressel P, Ma C, Bradfield LA, Killcross S, McNally GP. Punishment insensitivity emerges from impaired contingency detection, not aversion insensitivity or reward dominance. eLife. 2019:8:e52765.

78. Glessner JT, et al. Autism genome-wide copy number variation reveals ubiquitin and neuronal genes. Nature. 2009:459(7246):569-73.

79. Luo J, Norris RH, Gordon SL, Nithianantharajah J. Neurodevelopmental synaptopathies: insights from behaviour in rodent models of synapse gene mutations. Prog Neuropsychopharmacol Biol Psychiatry. 2018;84(Pt B):424-39. 
80. Varoqueaux F, et al. Neuroligins determine synapse maturation and function. Neuron. 2006;51(6):741-54.

81. Heath CJ, Bussey TJ, Saksida LM. Motivational assessment of mice using the touchscreen operant testing system: effects of dopaminergic drugs. Psychopharmacology. 2015;232(21-22):4043-57.

82. Rawat A, Guo J, Renoir T, Pang TY, Hannan AJ. Hypersensitivity to sertraline in the absence of hippocampal 5-HT1AR and 5-HTT gene expression changes following paternal corticosterone treatment. Environ Epigenet. 2018;4(2):dvy015.

83. Machado JAF, Parente PMDC, \& Santos Silva JMC QREG2: Stata module to perform quantile regression with robust and clustered standard errors. Statistical Software Components, Boston College Department of Economics. 2011:S457369.

84. Rescorla RA, Wagner AR. A theory of Pavlovian conditioning: Variations in the effectiveness of reinforcement and nonreinforcement. In Black $A H$ Prokasy WF (editors) Classical Conditioning II: Current Research and Theory. New York: Appleton-Century-Crofts. 1972:64-99.

85. Beraldo FH, et al. MouseBytes, an open-access high-throughput pipeline and database for rodent touchscreen-based cognitive assessment. eLife. 2019;8:e49630

86. Luo J, Tan JM, \& Nithianantharajah J. Associative learning and motivation differentially requires neuroligin-1 at excitatory synapses. bioRxiv. 2020 https://doi.org/10.1101/2020.01.01.890798

\section{Publisher's Note}

Springer Nature remains neutral with regard to jurisdictional claims in published maps and institutional affiliations.

Ready to submit your research? Choose BMC and benefit from:

- fast, convenient online submission

- thorough peer review by experienced researchers in your field

- rapid publication on acceptance

- support for research data, including large and complex data types

- gold Open Access which fosters wider collaboration and increased citations

- maximum visibility for your research: over $100 \mathrm{M}$ website views per year

At BMC, research is always in progress.

Learn more biomedcentral.com/submissions 